\title{
Stochastic hybrid systems: a modeling and stability theory tutorial
}

\author{
Andrew R. Teel and João P. Hespanha
}

\begin{abstract}
Stochastic hybrid systems are driven by random processes and have states that can both flow continuously and jump instantaneously. Many classes of stochastic hybrid systems, with different modeling strengths, have been considered in the literature. In this tutorial paper we first consider perhaps the simplest class of stochastic hybrid systems: those that admit unique solutions and that do not permit state conditions that force jumps. Several examples are given to illustrate the utility of this simple modeling class and Lyapunov-based sufficient conditions for various stability properties are given. The second half of the tutorial addresses a recent, more general stochastic hybrid systems modeling framework that permits state conditions to trigger jumps and that allows for non-unique solutions, via stochastic differential and difference inclusions and possibly overlapping flow and jump sets. Examples are provided to show the relevance of models that admit nonunique solutions and forced jumps. Lyapunov-based sufficient conditions for various stability properties for this class of stochastic hybrid systems are also provided.
\end{abstract}

\section{INTRODUCTION}

This paper constitutes a tutorial on certain approaches to modeling and analyzing stability properties of stochastic hybrid systems. The latter refers to systems with states that sometimes change continuously (flow) and sometimes change instantaneously (jump), with each type of evolution possibly driven by a random process. The tutorial focuses on two extreme modeling cases. It starts by discussing models with unique solutions, deterministic flows, and deterministic jumps where the timing of the jumps is random; it concludes by formulating models that admit non-unique solutions and include stochastic flows and stochastic jumps, allowing jumps that are triggered by the value of the state. A basic reference for more information pertaining the first part of the tutorial is [1]. References for more information pertaining to the second part of the tutorial include [2], [3], [4], [5], [6]. For both parts of the tutorial, examples are presented that fit the modeling structure, the solution concept is described, useful stability definitions are provided, Lyapunov-based sufficient conditions for the stability properties are stated, and stability is addressed for the examples using the provided analysis tools.

There are many results on stability theory from the stochastic hybrid systems literature that are not discussed in this tutorial. The paper [7] contains a recent survey of such results. As indicated there, some of the foundational frameworks for stochastic hybrid systems include piecewise

The authors are with the Electrical and Computer Engineering Department, University of California, Santa Barbara, CA 93106-9560. teel@ece.ucsb.edu, hespanha@ece.ucsb.edu. Research supported in part by AFOSR grant FA9550-15-1-0155, NSF grant ECCS-1232035, NSF Grant CNS-1329650, and NSF Grant EF-1137835. deterministic Markov processes [8], [9], diffusions driven by Lévy processes [10], Markov jump systems [11], hybrid switching diffusions [12], [13], and stochastic hybrid automata [14].

Rather than surveying such results, the aim of this tutorial is to provide exposure to two extreme classes of stochastic hybrid systems. An understanding of the basic results associated with these classes may inspire the reader to delve more deeply into the multi-faceted literature on stochastic hybrid systems, exploring models that reside somewhere between these two extremes.

Sections II-V below comprise the first part of the tutorial. After discussing a solution concept for a class of deterministic hybrid systems at the start of Section II, stochasticity is added. Examples that are covered by this modeling framework are presented in Section III. A first pass at stability theory definitions, appropriate for solutions with sample paths that have at most one jump at a time, appears in Section IV. Lyapunov-based sufficient conditions for these properties also appear in Section IV, and in Section V they are applied to the examples.

Sections VI-XIII comprise the second part of the tutorial. The structure of this part is similar to the structure of the first part. A formal model is presented in Section VI and examples fitting the modeling framework are given in Section VII. The solution concept, an extension of the solution concept for non-stochastic hybrid inclusions as found in [15] for example, is described in Section VIII. Stability definitions, appropriate for solutions with sample paths defined on hybrid time domains, appear in Section IX. Lyapunov-based sufficient conditions for these properties appear in Section $\mathrm{X}$, and in Section XII they are applied to the examples. In between these two sections, additional results that have been developed for the special case of non-stochastic flows appear in Section XI. A discussion of some open problems, including extending the results of Section XI to the case of flows modeled by stochastic differential inclusions, can be found in Section XIII. In contrast to the first part of the tutorial, the second part of the tutorial makes extensive use of set-valued analysis, a basic reference for which is [16]. As such, the solution concept and stability definitions are expressed in non-standard terms; on the other hand, it can be argued that the terms in which these items are expressed is natural. In any case, the second part of this tutorial makes an attempt to present these new concepts in a streamlined manner. 


\section{Stochastic Hybrid Systems}

In this section we focus our attention on hybrid systems (HS) whose state $x(t), t \geqslant 0$ takes values in $\mathbb{R}^{n}$. In practice, the state vector often has components that take values in discrete sets, but that fact will not be important for most of the discussion in this tutorial so we will not bother to distinguish between those components of the state that take continuous values and those that take discrete values. The reader is referred to [1] for the more general case that includes a HS state with both continuous and discrete components.

To facilitate the introduction of our stochastic hybrid systems model, we start with a deterministic model and subsequently add stochasticity to its solution.

\section{A. Deterministic Hybrid Systems}

A simple deterministic hybrid systems (DHSs) model requires a locally Lipschitz vector field $f: \mathbb{R}^{n} \rightarrow \mathbb{R}^{n}$ that defines the evolution of the state $x(t)$; a family of reset maps $\left\{\phi_{\ell}: \mathbb{R}^{n} \rightarrow \mathbb{R}^{n}, \ell \in \mathcal{L}\right\}$ that characterizes how the resets or impulses change the system's state; and a family of guards $\left\{g_{\ell}: \mathbb{R}^{n} \rightarrow \mathbb{R}, \ell \in \mathcal{L}\right\}$ that defines when resets or impulses take place. The families of reset maps and guards are parameterized by the parameter $\ell \in \mathcal{L}$ so that the guard $g_{\ell}$ corresponds to the reset map $\phi_{\ell}$.

A solution to this DHS, starting at an initial condition $x(0)=x_{0} \in \mathbb{R}^{n}$, is constructed as follows:

1) Set $k=0, t_{0}=0$.

2) Solve the initial-value problem $z\left(t_{k}\right)=x\left(t_{k}\right), \dot{z}=$ $f(z), \forall t \geqslant t_{k}$; and let $\left[t_{k}, T\right)$ denote its maximum interval of existence.

3) If any one of the $g_{\ell}(z(t))$ is non-negative at time $t_{k}$ or two or more of the $g_{\ell}(z(t))$ became nonnegative simultaneously in the interval $\left(t_{k}, T\right)$, then this procedure fails.

4) If a single $g_{\ell}(z(t)), \ell \in \mathcal{L}$ becomes non-negative in the interval $\left(t_{k}, T\right)$, i.e.,

$$
t_{k+1}:=\inf \left\{t \in\left(t_{k}, T\right): \exists \ell \in \mathcal{L}, g_{\ell}(z(t)) \geqslant 0\right\}
$$

belongs to the (open) interval $\left(t_{k}, T\right)$ and $\exists \ell_{k} \in \mathcal{L}$ such that

$$
g_{\ell_{k}}\left(z\left(t_{k+1}\right)\right) \geqslant 0, \quad g_{\ell}\left(z\left(t_{k+1}\right)\right)<0, \quad \forall \ell \neq \ell_{k} ;
$$

then define the DHS state in the interval $\left[t_{k}, t_{k+1}\right)$ to be

$$
x(t)=z(t), \quad \forall t \in\left[t_{k}, t_{k+1}\right)
$$

and at the time $t_{k+1}$ to be

$$
x\left(t_{k+1}\right)=\phi_{\ell_{k}}\left(z\left(t_{k+1}\right)\right)
$$

Increment the integer $k$ and go back to 2 .

5) If none of the $g_{\ell}(z(t)), \ell \in \mathcal{L}$ becomes non-negative in the interval $\left[t_{k}, T\right)$, then define the DHS state in the interval $\left[t_{k}, T\right)$ to be

$$
x(t)=z(t), \quad \forall t \in\left[t_{k}, T\right)
$$

and terminate.

We call the $t_{k}$ reset times and this solution is defined up to a maximal time $T_{\max }$ that can be either equal to $T$ if the construction terminate through 5 or $\sup _{k} t_{k}$ if it does not terminate.

Richer DHS models can be found in the literature. In particular, the model above lacks non-determinism in that an initial condition leads to uniqueness of solution, which simplifies the introduction of stochasticity. However, nondeterminism can be combined with stochasticity as we shall see starting in Section VI.

\section{B. Time-Triggered Stochastic Hybrid Systems}

In Section II-A, the reset times correspond to the times at which one of the guards $g_{\ell}$ becomes non-negative. In Time-Triggered Stochastic Hybrid Systems (TTSHS), the reset times are random variables with the property that the interreset intervals $\mathbf{h}_{k}:=\mathbf{t}_{k+1}-\mathbf{t}_{k}, \forall k \in \mathbb{Z}_{\geqslant 0}$ are independent and identically distributed random variables.

A TTSHS model requires three ingredients: a locally Lipschitz vector field $f: \mathbb{R}^{n} \rightarrow \mathbb{R}^{n}$ that defines the evolution of the state $x(t)$; a family of reset maps $\left\{\phi_{\ell}: \mathbb{R}^{n} \rightarrow \mathbb{R}^{n}, \ell \in\right.$ $\mathcal{L}\}$ that characterizes how the resets or impulses change the system's state; and a family of reset-time distributions $\left\{\mu^{\ell}: \ell \in \mathcal{L}\right\}$ that defines when resets or impulses take place. Also here, the families of reset maps and reset-time distributions are parameterized by the same parameter $\ell \in \mathcal{L}$.

The construction of a solution to this TTSHS is similar to that of a DHS, but with a different selection method for the reset times. In particular, the steps $2-5$ should be replaced by:

2') Solve the initial-value problem $\mathbf{z}\left(\mathbf{t}_{k}\right)=\mathbf{x}\left(\mathbf{t}_{k}\right), \dot{\mathbf{z}}=$ $f(\mathbf{z}), \forall t \geqslant \mathbf{t}_{k} ;$ and let $\left[\mathbf{t}_{k}, \mathbf{T}\right)$ denote its maximum interval of existence.

3') Draw independent random numbers $\mathbf{h}_{k}^{\ell}, \ell \in \mathcal{L}$ with distributions $\mu^{\ell}$. If any of the $\mathbf{h}_{k}^{\ell}$ is equal to zero or more than one of the $\mathbf{h}_{k}^{\ell}$ is equal to $\mathbf{h}_{k}:=\min _{\ell \in \mathcal{L}} \mathbf{h}_{k}^{\ell}$ then this procedure fails. Otherwise set

$$
\mathbf{t}_{k+1}:=\mathbf{t}_{k}+\mathbf{h}_{k} .
$$

4') If $\mathbf{t}_{k+1} \in\left(\mathbf{t}_{k}, \mathbf{T}\right)$ and a single $\mathbf{h}_{k}^{\ell}$ is equal to $\mathbf{h}_{k}$, i.e., $\exists \ell_{k} \in \mathcal{L}$ such that

$$
\mathbf{h}_{k}^{\ell_{k}}=\mathbf{h}_{k}, \quad \mathbf{h}_{k}^{\ell}>\mathbf{h}_{k}, \quad \forall \ell \neq \ell_{k} ;
$$

then define the TTSHS state in the interval $\left[\mathbf{t}_{k}, \mathbf{t}_{k+1}\right)$ to be

$$
\mathbf{x}(t)=\mathbf{z}(t), \quad \forall t \in\left[\mathbf{t}_{k}, \mathbf{t}_{k+1}\right)
$$

and at the time $\mathbf{t}_{k+1}$ to be

$$
\mathbf{x}\left(\mathbf{t}_{k+1}\right)=\phi_{\boldsymbol{\ell}_{k}}\left(\mathbf{z}\left(\mathbf{t}_{k+1}\right)\right)
$$

Increment the integer $k$ and go back to 2 '.

5') If $\mathbf{t}_{k+1} \geqslant \mathbf{T}$, then define the TTSHS state in the interval $\left[\mathbf{t}_{k}, \mathbf{T}\right)$ to be

$$
\mathbf{x}(t)=\mathbf{z}(t), \quad \forall t \in\left[\mathbf{t}_{k}, \mathbf{T}\right)
$$


and terminate.

Following the terminology introduced for the DHSs, we call the random times $\mathbf{t}_{k}$ reset times and the solution is defined up to a maximal time $\mathbf{T}_{\max }$ that is equal to $\mathbf{T}$ if the construction terminates through $5^{\prime}$ or is equal to $\sup _{k} \mathbf{t}_{k}$ if it does not terminate.

To make sure that the procedure above does not fail in step 3', one should require that $\mathbb{P}\left(\mathbf{h}_{k}^{\ell}=0\right)=0$ for every $\ell \in \mathcal{L}, k \in \mathbb{Z}_{\geqslant 0}$ (so that we do not get $\mathbf{h}_{k}^{\ell}$ equal to zero) and also that $\mathbb{P}\left(\mathbf{h}_{k}^{\ell_{1}}=\mathbf{h}_{k}^{\ell_{2}}\right)=0$ for every $\ell_{1} \neq \ell_{2}, k \in \mathbb{Z}_{\geqslant 0}$ (so that we do not get more than one of the $\mathbf{h}_{k}^{\ell}$ is equal to $\left.\mathbf{h}_{k}:=\min _{\ell \in \mathcal{L}} \mathbf{h}_{k}^{\ell}\right)$.

\section{State-Driven Stochastic Hybrid Systems}

For the TTSHS model above, the distributions of the interreset times are not allowed to depend on the current state value. However, in State-Driven Stochastic Hybrid Systems $(S D S H S)$ the state $\mathbf{x}(t)$ plays a role in the selection of the reset times.

A SDSHS model also requires three ingredients: a locally Lipschitz vector field $f: \mathbb{R}^{n} \rightarrow \mathbb{R}^{n}$ that defines the evolution of the state $x(t)$; a family of reset maps $\left\{\phi_{\ell}: \mathbb{R}^{n} \rightarrow \mathbb{R}^{n}, \ell \in\right.$ $\mathcal{L}\}$ that characterizes how the jumps or impulses change the system's state; and a family of transition intensities $\left\{\lambda_{\ell}\right.$ : $\left.\mathbb{R}^{n} \rightarrow[0, \infty), \ell \in \mathcal{L}\right\}$ that defines when jumps or impulses take place. Each transition intensity $\lambda_{\ell}, \ell \in \mathcal{L}$ determines the "instantaneous" probability of the reset $\phi_{\ell}$, in the sense that the probability that the state will be reset by $\phi_{\ell}$ during the "elementary" interval $(t, t+d t]$ is given by $\lambda_{\ell}(\mathbf{x}(t)) d t$. By an "elementary" time interval, we mean that this statement holds in the limit as $d t \downarrow 0^{+}$. One can thus regard $\lambda_{\ell}(\mathbf{x}(t))$ as the "instantaneous" average number of resets $\phi_{\ell}$ per unit of time.

To construct a solution to this SDSHS, one follows similar steps to those in Section II-A for a DHS, but with steps 2-5 replaced by:

2") Solve the initial-value problem $\mathbf{z}\left(\mathbf{t}_{k}\right)=\mathbf{x}\left(\mathbf{t}_{k}\right), \dot{\mathbf{z}}=$ $f(\mathbf{z}), \forall t \geqslant \mathbf{t}_{k}$; and let $\left[\mathbf{t}_{k}, \mathbf{T}\right)$ denote its maximum interval of existence.

3") Draw independent random numbers $\mathbf{h}_{k}^{\ell}, \ell \in \mathcal{L}$ with standard exponential distributions (unit mean and variance).

4") If one of the integrals

$$
\mathbf{m}_{k}^{\ell}(t):=\int_{\mathbf{t}_{k}}^{t} \lambda_{\ell}(\mathbf{z}(s)) d s, \quad \forall t \in\left[\mathbf{t}_{k}, \mathbf{T}\right), \ell \in \mathcal{L}
$$

reaches $\mathbf{h}_{k}^{\ell}, \ell \in \mathcal{L}$ in the interval $\left(\mathbf{t}_{k}, \mathbf{T}\right)$, i.e., if

$$
\mathbf{t}_{k+1}:=\inf \left\{t \in\left(\mathbf{t}_{k}, \mathbf{T}\right): \exists \ell \in \mathcal{L}, \mathbf{m}_{k}^{\ell}(t) \geqslant \mathbf{h}_{k}^{\ell}\right\}
$$

belongs to the (open) interval $\left(\mathbf{t}_{k}, T\right)$ and $\exists \ell_{k} \in \mathcal{L}$ such that $\mathbf{m}_{k}^{\ell_{k}}(t) \geqslant \mathbf{h}_{k}^{\ell_{k}}$; then define the SDSHS state in the interval $\left[\mathbf{t}_{k}, \mathbf{t}_{k+1}\right]$ according to (2)-(3). Increment the integer $k$ and go back to 2 ".

5") Otherwise, define the SDSHS state in the interval $\left[\mathbf{t}_{k}, \mathbf{T}\right)$ according to (4) and terminate.
We assume that the transition intensity maps $x \mapsto \lambda_{\ell}(x), \ell \in$ $\mathcal{L}$ are continuous (or at least measurable) to make sure that the integrals in (5) are well defined; and that the transition intensities $\lambda_{\ell}(x), \ell \in \mathcal{L}$ and the reset maps $\phi_{\ell}(x), \ell \in \mathcal{L}$ are locally bounded in $x$, in the sense that, for every bounded set $\mathcal{B} \subset \mathbb{R}^{n}$, there exist constants $L_{\mathcal{B}}, F_{\mathcal{B}}$ such that

$$
\lambda_{\ell}(x) \leqslant L_{\mathcal{B}}, \quad\left\|\phi_{\ell}(x)\right\| \leqslant F_{\mathcal{B}}, \quad, \forall x \in \mathcal{B},
$$

to make sure that, if the state $\mathrm{x}$ remains inside a bounded set, then $\mathbf{T}_{\max }=\infty$. This last assumption is not required for the construction of the solution, but it is instrumental in establishing global existence of solutions.

When constructing solutions to SDSHS, the $\mathbf{h}_{k}^{\ell}, \ell \in \mathcal{L}$ are all positive with probability one and one does not have to worry about $\mathbf{t}_{k+1}$ in step 4 " being equal to $\mathbf{t}_{k}$. The probability of multiple $\mathbf{m}_{k}^{\ell}$ reaching the $\mathbf{h}_{k}^{\ell}$ simultaneously is also zero, so $\ell_{k}$ in step 4 " is well defined with probability one.

D. Emulating Time-Triggered Stochastic Hybrid Systems with State-Driven Stochastic Hybrid Systems

It turns out that TTSHSs can be emulated by appropriately constructed SDSHSs when the reset-time distributions $\mu^{\ell}(\cdot)$ have probability density functions $f^{\ell}:[0, \infty) \rightarrow[0, \infty)$, i.e.,

$$
\begin{aligned}
& F^{\ell}(h):=\int_{[0, h]} \mu^{\ell}(d s)=\int_{0}^{h} f^{\ell}(s) d s, \\
& \forall h \in\left[0, T^{\ell}\right), \ell \in \mathcal{L},
\end{aligned}
$$

where $T^{\ell}:=\sup \left\{h \in \mathbb{R}: F^{\ell}(h)<1\right\} \in \mathbb{R} \cup\{\infty\}$. To construct a SDSHS that emulates the TTSHS, we augment the state of the TTSHS with a timer variable

$$
\boldsymbol{\tau}(t)=t-\mathbf{t}_{k}, \quad \forall t \geqslant 0,
$$

leading to a SDSHS with state-space $\mathbb{R}^{n} \times \mathbb{R}$. The differential equation for $\tau$ is

$$
\dot{\tau}=1
$$

and we build the reset maps $\phi_{\ell}: \mathbb{R}^{n} \times \mathbb{R} \rightarrow \mathbb{R}^{n} \times \mathbb{R}$ to set $\boldsymbol{\tau}=0$ at each reset time $\mathbf{t}_{k}$. The transition intensities $\lambda_{\ell}(x, \tau)$ associated with these resets are given by the hazard rate of the reset-time distributions $\mu^{\ell}$ :

$$
\lambda_{\ell}(x, \tau):=\frac{f^{\ell}(\tau)}{1-F^{\ell}(\tau)}, \quad \forall x \in \mathbb{R}^{n}, \tau \in\left[0, T^{\ell}\right),
$$

which turn out to be equal to the probability that a random timer $\tau$ with distribution $\mu^{\ell}$ will fire in an elementary interval $[\tau, \tau+d \tau)$, given that it has not yet fired at time $\tau$. One can show that this construction guarantees that the (conditional) distribution of $\mathbf{t}_{k+1}$ given $\mathbf{t}_{k}$ is equal to

$$
\begin{aligned}
\mathbb{P}\left(\mathbf{t}_{k+1}>T \mid \mathbf{t}_{k}\right)= & \prod_{\ell \in \mathcal{L}} \int_{\left(T-\mathbf{t}_{k}, \infty\right)} f^{\ell}(s) d s \\
& =\mathbb{P}\left(\mathbf{h}_{k}^{\ell}>T-\mathbf{t}_{k}, \forall \ell \in \mathcal{L} \mid \mathbf{t}_{k}\right)
\end{aligned}
$$

which is precisely what one would get for the corresponding TTSHS [1]. 
Representing TTSHSs as SDSHSs enables one to combine in the same SHS both time-triggered and state-driven resets. The approach outlined above applies only when the resettime distributions do not have point masses. This limitation does not appear the models presented starting in Section VI.

\section{EXAMPLES}

\section{A. Feedback control using remote sensors}

Consider a feedback control system in which the process output vector $y$ is measured by a sensor that is located far from the controller and communicates with it through a shared network, as shown in Figure 1. The sensor samples the

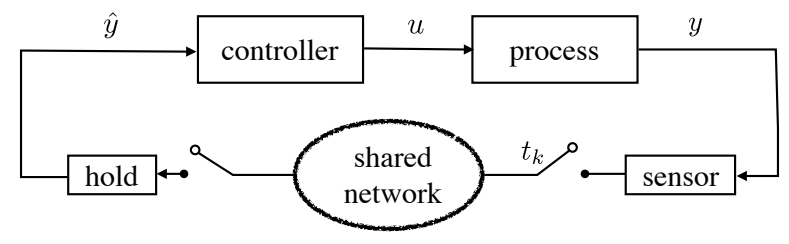

Fig. 1. Feedback loop with a shared network between sensor and controller.

process output $y$ at times $\mathbf{t}_{k}, k \in \mathbb{Z}_{\geqslant 0}$ and sends the value of $y$ at times $\mathbf{t}_{k}$ to the controller. Between sampling times, the controller simply holds the values of the last measurement received, leading to a mismatch between the process output $y(t)$ and the controller input $\hat{y}(t)$. For the following linear process and controller models,

$$
\begin{aligned}
\dot{x}_{P} & =A_{P} x_{P}+B_{P} u, & \dot{x}_{C} & =A_{C} x_{C}+B_{C} \hat{y} \\
y & =C_{P} x_{P}, & u & =C_{C} x_{C},
\end{aligned}
$$

the system dynamics can be written as

$$
\dot{x}=A x, \quad x:=\left[\begin{array}{c}
x_{P} \\
x_{C} \\
\hat{y}
\end{array}\right], \quad A:=\left[\begin{array}{ccc}
A_{P} & B_{P} C_{C} & 0 \\
0 & A_{C} & B_{C} \\
0 & 0 & 0
\end{array}\right],
$$

between sampling times; and at each sampling time $\mathbf{t}_{k} \in$ $\mathbb{Z}_{\geqslant 1}$

$$
x\left(\mathbf{t}_{k}\right):=J x^{-}\left(\mathbf{t}_{k}\right), \quad J:=\left[\begin{array}{ccc}
I & 0 & 0 \\
0 & I & 0 \\
C_{P} & 0 & 0
\end{array}\right]
$$

which corresponds to continuity for $x_{P}$ and $x_{C}$ and, at the sampling times, $\hat{y}\left(\mathbf{t}_{k}\right)$ is set equal to $y\left(\mathbf{t}_{k}\right)=C_{P} x_{P}\left(\mathbf{t}_{k}\right)$.

Typically, the sensor samples the process output periodically with the sampling time equal to a constant $T_{s}>0$, but when measurements are communicated through a shared network, transmission fails if the network is busy and the sensor may need to wait until some time $\mathbf{t}_{k}$, at which time the sensor sends the current output $y\left(\mathbf{t}_{k}\right)$. In this case, the interval $\mathbf{h}_{k}:=\mathbf{t}_{k+1}-\mathbf{t}_{k}$ becomes a random variable due to the stochastic nature of the wait. The resulting system can be modeled using a TTSHS with the vector field (7), the reset map

$$
\phi(x)=J x,
$$

and the reset-time distribution $\mu(\cdot)$ of the random variables $\mathbf{h}_{k}:=\mathbf{t}_{k+1}-\mathbf{t}_{k}$.

\section{B. Estimation through a packet-switched network}

Consider the setup shown in Figure 2, where one wants to construct a remote estimate for the state of a process, by sending state measurements through a packet-switched shared network. The process is the following linear system

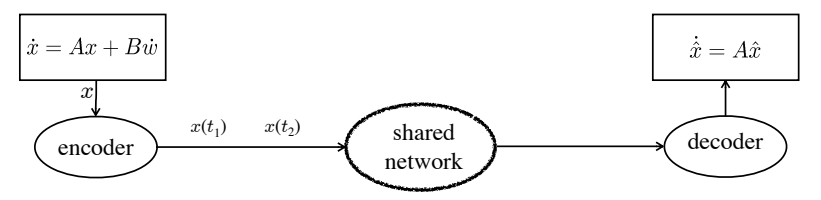

Fig. 2. Estimation through a packet-switched network in Example III-B.

$$
\dot{\mathbf{x}}=A \mathbf{x}+b \dot{\mathbf{w}}, \quad \mathbf{x} \in \mathbb{R}^{n},
$$

where $\mathbf{w}$ denotes a scalar Brownian motion process. To focus our attention on SHSs whose dynamics are ordinary differential equations (ODEs), we employ the mathematical trick of approximating the stochastic differential equation (SDE) (8) by a random walk that can be easily modeled by an ODE with stochastic impulses: It turns out that the solutions to (8) can be obtained as the limit $\epsilon \downarrow 0^{+}$of the solutions to a SDSHS with continuous dynamics

$$
\dot{\mathrm{x}}=A \mathrm{x}
$$

and two reset maps

$$
\phi_{1}(x):=x+\sqrt{\epsilon} b, \quad \phi_{2}(x):=x-\sqrt{\epsilon} b, \quad \forall x \in \mathbb{R}^{n},
$$

with the same transition intensities

$$
\lambda_{1}(x)=\lambda_{2}(x):=\frac{1}{2 \epsilon}, \quad \forall x \in \mathbb{R}^{n} .
$$

The encoder samples the state at random times $\mathbf{t}_{k}, k \in \mathbb{Z}_{\geqslant 0}$ and sends the sampled states $\mathbf{x}\left(\mathbf{t}_{k}\right)$ to a decoder that builds the remote estimate $\hat{\mathbf{x}}$ of the true state $\mathbf{x}$. Between sample times, the decoder uses an open-loop state estimator of the form

$$
\dot{\hat{\mathbf{x}}}=A \hat{\mathbf{x}}, \quad \forall t \in\left[\mathbf{t}_{k}, \mathbf{t}_{k+1}\right),
$$

and, whenever a sample $\mathbf{x}\left(\mathbf{t}_{k}\right)$ is received, it resets the estimate $\hat{\mathbf{x}}\left(\mathbf{t}_{k}\right)$ to the value received. We are interested in an encoder that generates the $\mathbf{t}_{k}$ stochastically, based on the current value of the state estimation error $\mathbf{e}:=\mathrm{x}-\hat{\mathbf{x}}$. The rationale for this is that the encoder should increase the sampling rate when the estimation error is large to correct this error as fast as possible by sending more measurements, whereas there is little gain in sending measurements when the error is already very small.

Between "event" times, the resulting estimation error evolves according to

$$
\dot{\mathbf{e}}=A \mathbf{e},
$$


but this dynamics is perturbed by two types of events: jumps in $\mathbf{x}$ due to the noise process (9) that lead to resets of the form

$$
\begin{aligned}
& \mathbf{e}\left(\mathbf{t}_{k}\right)=\phi_{1}\left(\mathbf{e}^{-}\left(\mathbf{t}_{k}\right)\right):=\mathbf{e}^{-}\left(\mathbf{t}_{k}\right)+\sqrt{\epsilon} b \\
& \mathbf{e}\left(\mathbf{t}_{k}\right)=\phi_{2}\left(\mathbf{e}^{-}\left(\mathbf{t}_{k}\right)\right):=\mathbf{e}^{-}\left(\mathbf{t}_{k}\right)-\sqrt{\epsilon} b
\end{aligned}
$$

both with intensity $1 / 2 \epsilon$; and resets of the estimate $\hat{\mathbf{x}}$ due to the update of the estimate that leads to resets of the form

$$
\mathbf{e}\left(\mathbf{t}_{k}\right)=\phi_{3}\left(\mathbf{e}^{-}\left(\mathbf{t}_{k}\right)\right):=0,
$$

with an intensity that depends on the current state estimation error e. These error dynamics can thus be represented by a SDSHS with continuous dynamics given by (11) and three reset maps: two of them are used to model the noise process and correspond to the resets in (12) and the associated transition intensities (10), whereas the third models the sampling of the state and corresponds to the reset in (14) and an intensity $\lambda(\mathbf{e})$ that can be viewed as a design parameter.

\section{STABILITY THEORY}

\section{A. Definitions}

The stability of a stochastic process is typically characterized by two types of conditions: sample-path stability notions address the behavior of individual sample paths of the stochastic process, whereas mean or aggregate notions of stability address the average behavior of the system trajectories.

Sample-path stability notions are typically qualified by the suffix "in probability." A SHS (either time-triggered or state-driven) with state $\mathbf{x}:\left[0, \mathbf{T}_{\max }\right) \rightarrow \mathbb{R}^{n}$ is said to be (Lyapunov) stable in probability if it is globally defined with probability one for every $\epsilon, \rho>0$, there exists a $\delta>0$ such that, for every initial condition $\mathbf{x}(0)=x_{0} \in \mathbb{R}^{n}$,

$$
\left\|x_{0}\right\| \leqslant \delta \Rightarrow \mathbb{P}(\exists t \in[0, \infty):\|\mathbf{x}(t)\|>\rho) \leqslant \epsilon ;
$$

and (Lyapunov) asymptotically stable in probability if it is (Lyapunov) stable in probability and $\mathbf{x}(t)$ converges to zero with probability one, i.e.,

$$
\mathbb{P}\left(\lim _{t \rightarrow \infty} \mathbf{x}(t)=0\right)=1 .
$$

Aggregate or mean notions of stability require the selection of a non-negative function $W: \mathbb{R}^{n} \rightarrow[0, \infty)$ and the SHS is said to be mean- $W$ stable if it is globally defined with probability one and, for every initial condition $\mathrm{x}(0)=x_{0} \in$ $\mathbb{R}^{n}$,

$$
\mathbb{E}[W(\mathbf{x}(t))]<\infty, \quad \forall t \geqslant 0
$$

stochastically mean-W stable if it is globally defined with probability one and, for every initial condition $\mathrm{x}(0)=x_{0} \in$ $\mathbb{R}^{n}$,

$$
\int_{0}^{\infty} \mathbb{E}[W(\mathbf{x}(t))] d t<\infty
$$

asymptotically mean-W stable if it is globally defined with probability one and, for every initial condition $\mathbf{x}(0)=x_{0} \in$ $\mathbb{R}^{n}$,

$$
\lim _{t \rightarrow \infty} \mathbb{E}[W(\mathbf{x}(t))]=0 ;
$$

and exponentially mean- $W$ stable if it is globally defined with probability one and there exist constants $c, \lambda>0$ such that, for every initial condition $\mathbf{x}(0)=x_{0} \in \mathbb{R}^{n}$,

$$
\mathbb{E}[W(\mathbf{x}(t))] \leqslant c e^{-\lambda t} W\left(x_{0}\right), \quad \forall t \geqslant 0 .
$$

When $W(x)=\|x\|^{2}, \forall x \in \mathbb{R}^{n}$, we simply say that the processes are mean-square stable, without reference to $W$. When some components of the SHS state take values in discrete sets, the vector $\mathbf{x}$ that appears in the definitions above is often just the continuous portion of the state, as one often does care whether or not the discrete component of the state converges to a specific value. This type of stability is addressed explicitly in Section IX, in the context of stochastic hybrid inclusions, where stability of sets is considered.

\section{B. Analysis of Time-Triggered SHSs}

Fairly complete stability results are available for TTSHSs with a linear vector field

$$
\dot{\mathbf{x}}=A \mathbf{x}
$$

and a linear reset map

$$
\phi(x):=J x, \quad \forall x \in \mathbb{R}^{n}
$$

associated with a reset-time distribution $\mu$. Necessary and sufficient conditions for the stability of the TTSHS defined by $16-(17)$ depend on the type of stochastic stability considered, as expressed by the result below.

Theorem 1 (Stability of a Linear TTSHS [17], [1]):

Consider a TTSHS defined by the vector field (16), the reset map (17), and the reset-time distribution $\mu$. Further assume that

$$
\begin{aligned}
\sigma\left(\sum_{i} \mu\left(\left\{b_{i}\right\}\right)\left(J e^{A b_{i}}\right)^{\prime} \otimes\left(J e^{A b_{i}}\right)\right)<1, \\
\exists \lambda>\lambda^{*}: \quad \int_{0}^{\infty} e^{2 \lambda h} \mu(d h)<\infty,
\end{aligned}
$$

where $\sigma(M)$ denotes the spectral radius of the matrix $M$, the summation inside $\sigma(\cdot)$ is taken over any point masses $b_{i}$ of the reset-time distribution $\mu$, and $\lambda^{*}$ denotes the real part of the eigenvalue of $A$ with largest real part for which one of the corresponding generalized eigenvectors is not in the kernel of $J$, i.e.,

$$
\begin{aligned}
\lambda^{*}:=\max \left\{\mathbb{R}[\lambda]:(A-\lambda I)^{k} x=0,\right. & \\
& \left.J x \neq 0, k \in \mathbb{Z}_{\geqslant 1}, x \in \mathbb{R}^{n}, \lambda \in \mathbb{C}\right\} .
\end{aligned}
$$

(i) This system is stochastically mean-square stable if and only if there exists a positive definite matrix $P=P^{\prime}>0$ such that

$$
L(P)<P, \quad L(P):=\int_{0}^{\infty} e^{A^{\prime} h} J^{\prime} P J e^{A h} \mu(d h),
$$


and

$$
\int_{0}^{\infty} h^{2\left(m_{\mathbb{R}}(A)-1\right)} e^{2 \lambda_{\mathbb{R}}(A) h} r(h) d h<\infty,
$$

where $\lambda_{\mathbb{R}}(A)$ denotes the real part of the eigenvalue of $A$ with largest real part, $m_{\mathbb{R}}$ the dimension of the corresponding Jordan block, and $r(h):=\mathbb{P}\left(\mathbf{h}_{k}>h\right)=$ $\int_{(h, \infty)} \mu(d h)$ denotes the survivor function of $\mu$.

(ii) This system is asymptotically mean-square stable if and only if there exists a positive definite matrix $P=P^{\prime}>0$ such that (18) holds and

$$
\lim _{h \rightarrow \infty} h^{2\left(m_{\mathbb{R}}(A)-1\right)} e^{2 \lambda_{\mathbb{R}}(A) h} r(h)=0 .
$$

(iii) This system is exponentially mean-square stable if and only if there exists a positive definite matrix $P=P^{\prime}>0$ such that (18) holds and there exist constants $c, \alpha>0$ such that

$$
h^{2\left(m_{\mathbb{R}}(A)-1\right)} e^{2 \lambda_{\mathbb{R}}(A) h} r(h) \leqslant c e^{-\alpha h}, \quad \forall h \geqslant 0 .
$$

In essence, the existence of the positive definite matrix $P=P^{\prime}>0$ such that (18) holds is necessary for all three types of stability and guarantees that the value of the state $\mathbf{x}_{k}:=\mathbf{x}\left(\mathbf{t}_{k}\right)$ at the reset times converges to zero exponentially fast as $k \rightarrow \infty$. However, this does not suffice to prove stability. The conditions (19), (20), and (21) are needed to make sure that the inter-sampling behavior does not compromise stability.

The condition (18) is a Linear Matrix Inequality that can be easily verified numerically. The conditions (19), (20), and (21) are also easy to verify as they simply depend on the "least stable" eigenvalue of $A$.

\section{Analysis of State-Driven SHSs}

The extended generator of the SDSHS defined in Section II-C is an operator $L$ that maps a continuously differentiable function $V: \mathbb{R}^{n} \rightarrow \mathbb{R}$ into another function $L V: \mathbb{R}^{n} \rightarrow \mathbb{R}$ according to the rule:

$$
\begin{aligned}
L V(x) & :=\frac{\partial V(x)}{\partial x} f(x) \\
& +\sum_{\ell \in \mathcal{L}} \lambda_{\ell}(x)\left(V\left(\phi_{\ell}(x)\right)-V(x)\right), \quad \forall x \in \mathbb{R}^{n} .
\end{aligned}
$$

Under appropriate assumptions on the SDSHS and the function $V$, one can use this extended generator to compute derivatives along solutions to the SDSHS using the following formula

$$
\begin{aligned}
\frac{d \mathbb{E}[V(\mathbf{x}(t)) \mid \mathbf{x}(\tau)]}{d t} \stackrel{\text { wpo }}{=} \mathbb{E}[L V(\mathbf{x}(t)) \mid \mathbf{x}(\tau)], \\
\forall t \stackrel{\text { ae }}{\in}[\tau, \infty), \forall \tau \geqslant 0,
\end{aligned}
$$

where $\stackrel{\text { wpo }}{=}$ refers to the fact that this equality holds with probability one and $\stackrel{\text { ae }}{\in}$ refers to the fact that the derivative may not exist on a set of times $t$ with zero measure [1]. It is important to note that, even though the sample paths of the stochastic process $V(\mathrm{x}(t))$ are typically discontinuous because of the resets, the expected value of this stochastic process is not only continuous but, in fact, differentiable almost everywhere. We shall refer to (23) as the differential form of Dynkin's formula, which is the basis for establishing the following stability results for SDSHS.

Theorem 2 (Stability of a SDSHS [1]): Consider the SDSHS defined in Section II-C and a continuously differentiable, non-negative, and radially unbounded function $V$ : $\mathbb{R}^{n} \rightarrow \mathbb{R}$.

(i) If

$$
L V(x) \leqslant \rho V, \quad \forall x \in \mathbb{R}^{n}
$$

for some constant $\rho \geqslant 0$ then, for every initial condition $\mathbf{x}(0)=x_{0} \in \mathbb{R}^{n}$, the solution to the SDSHS is globally defined with probability one, i.e., $\mathbb{P}\left(\mathbf{T}_{\max }=\infty\right)=1$.

(ii) If (24) holds with $\rho=0$, i.e.,

$$
L V(x) \leqslant 0, \quad \forall x \in \mathbb{R}^{n}
$$

then, for every $m>0$ and every initial condition $\mathbf{x}(0)=$ $x_{0} \in \mathbb{R}^{n}$,

$$
\begin{aligned}
\mathbb{P}(\exists t \in[0, \infty): V(\mathbf{x}(t)) \geqslant \mu) & \\
& \stackrel{\text { wpo }}{\leqslant} \frac{V\left(x_{0}\right)}{\mu}, \quad \forall \mu \in(0, m] .
\end{aligned}
$$

(iii) If, in addition to (25), $V$ is positive definite, in the sense that

$$
V(x)>0, \quad \forall x \neq 0,
$$

then the SDSHS is stable in probability.

(iv) If, in addition to (25) and (27), $L V$ is negative definite, in the sense that there exists a function $\alpha$ of class $^{1} \mathcal{K}$ such that

$$
L V(x) \leqslant-\alpha(\|x\|), \quad \forall x \in \mathbb{R}^{n}
$$

then the SDSHS is asymptotically stable in probability.

Because of (23), the inequality (25) guarantees that the expected value of $V(\mathbf{x}(t))$ does not increase along solutions to the SDSHS. This would also be true if we required that

$$
\frac{\partial V(x)}{\partial x} f(x) \leqslant 0, \quad V\left(\phi_{\ell}(x)\right) \leqslant V(x), \quad \forall \ell \in \mathcal{L}, x \in \mathbb{R}^{n},
$$

where the left-hand side inequality essentially requires $V(\mathrm{x}(t))$ not to increase along the (deterministic) flows of $\dot{\mathbf{x}}=f(\mathbf{x})$ and the right-hand side inequality requires $V(\mathbf{x}(t))$ not to increase at each reset time. However, (25) is weaker than (28) because, for (25) to hold, the decrease needs to occur only in expected value, which permits some sample paths to exhibit strict increase on the value of $V(\mathbf{x}(t))$ along flows of $\dot{\mathbf{x}}=f(\mathbf{x})$ and/or strict increase on the value of $V\left(\mathbf{x}\left(\mathbf{t}_{k}\right)\right)$ at reset times.

\footnotetext{
${ }^{1}$ A function $\alpha:[0, \infty) \rightarrow[0, \infty)$ is of class $\mathcal{K}$ when $\alpha$ is continuous, strictly increasing, and $\alpha(0)=0$.
} 
While Theorem 2 was focused on establishing sample-path notions of stability, the following results concern aggregate stability notions.

Theorem 3 (Mean stability of a SDSHS): Consider the SDSHS defined in Section II-C and a continuously differentiable, non-negative, and radially unbounded function $V: \mathbb{R}^{n} \rightarrow \mathbb{R}$. Further assume that the solution to the SDSHS is globally defined with probability one ${ }^{2}$.

(i) If (25) holds, then the SDSHS is mean- $V$ stable and, for every initial condition $\mathbf{x}(0)=x_{0} \in \mathbb{R}^{n}$,

$$
\mathbb{E}[V(\mathbf{x}(t))] \leqslant V\left(x_{0}\right), \quad \forall t \geqslant 0 .
$$

(ii) If

$$
L V(x) \leqslant-W(x), \quad \forall x \in \mathbb{R}^{n},
$$

for a function $W: \mathbb{R}^{n} \rightarrow \mathbb{R}$ such that $W(x) \geqslant 0$, $\forall x \in \mathbb{R}^{n}$, then the SDSHS is stochastic mean- $W$ stable and, for every initial condition $\mathbf{x}(0)=x_{0} \in \mathbb{R}^{n}$,

$$
\int_{0}^{\infty} \mathbb{E}[W(\mathbf{x}(t))] \leqslant V\left(x_{0}\right), \quad \forall t \geqslant 0 .
$$

(iii) If

$$
L V(x) \leqslant-\rho V(x)+c, \quad \forall x \in \mathbb{R}^{n},
$$

for constants $c \geqslant 0, \rho>0$, then the SDSHS is mean- $V$ stable and, for every initial condition $\mathbf{x}(0)=x_{0} \in \mathbb{R}^{n}$,

$$
\mathbb{E}[V(\mathbf{x}(t))] \leqslant e^{-\rho t} V\left(x_{0}\right)+\frac{c}{\rho}\left(1-e^{-\rho t}\right), \quad \forall t \geqslant 0 .
$$

In case $c=0$, then the SDSHS is exponentially mean- $V$ stable.

(iv) If

$$
L V(x) \leqslant-W(x)+c, \quad \forall x \in \mathbb{R}^{n},
$$

for a constant $c \geqslant 0$ and a function $W: \mathbb{R}^{n} \rightarrow \mathbb{R}$ such that $W(x) \geqslant 0, \forall x \in \mathbb{R}^{n}$, then, for every initial condition $\mathbf{x}(0)=x_{0} \in \mathbb{R}^{n}$,

$$
\lim _{T \rightarrow \infty} \frac{1}{T} \int_{0}^{T} \mathbb{E}[W(\mathbf{x}(t))] d t \leqslant c .
$$

As with (deterministic or stochastic) differential equations, Lyapunov functions for SDSHSs may be hard to find, but this is not the case for the linear TTSHS considered in Theorem 1:

Theorem 4: Consider a TTSHS defined by the vector field (16), the reset map (17), and a reset-time distribution $\mu$ with a probability density function $f$. This system is uniformly exponentially mean-square stable if and only if there exists a positive definite matrix-valued function $P:[0, \infty) \rightarrow \mathbb{R}^{n \times n}$ and constants $c_{1}, c_{2}>0$ such that, for every positive definite matrices $S_{1}, S_{2} \in \mathbb{R}^{n}$,

$$
\begin{aligned}
& c_{1} I<P(\tau)<c_{2} I, \\
& \qquad \begin{array}{r}
\dot{P}(\tau)=-S_{1}-A^{\prime} P(\tau)-P(\tau) A \\
\quad-\lambda(\tau)\left(J^{\prime} P(0) J-P(\tau)+S_{2}\right),
\end{array}
\end{aligned}
$$

\footnotetext{
${ }^{2}$ This could be established, e.g., using (i) in Theorem 2.
}

$\forall \tau \in[0, T)$, where the intensity $\lambda(\tau)$ is obtained from the distribution $\mu$ using the procedure described in Section IID. In this case, $V(x, \tau):=x^{\prime} P(\tau) x, \forall x \in \mathbb{R}^{n}, \tau \geqslant 0$ is a continuously differentiable, positive definite, and radially unbounded function for which (30) holds with $c=0$ for the SDSHS whose construction is described in Section IID.

\section{THE EXAMPLES REVISITED}

\section{A. Feedback control using remote sensors}

Stability for this example can be determined directly from the conditions in Theorem 1.

\section{B. Estimation through a packet-switched network}

Going back to the example considered in Section III-B, the error dynamics of the state estimation error $\mathbf{e}$ can be modeled by the a SDSHS whose extended generator is given by

$$
\begin{aligned}
L V(e):=\frac{\partial V(e)}{\partial e} A e+\lambda(e)(V(0)-V(e)) \\
+\frac{1}{2 \epsilon}(V(e+\sqrt{\epsilon} b)-V(e))+\frac{1}{2 \epsilon}(V(e-\sqrt{\epsilon} b)-V(e)),
\end{aligned}
$$

$\forall e \in \mathbb{R}^{n}$. To establish global existence of solution, we consider the following Lyapunov function

$$
V_{\text {glob }}(e):=\|e\|^{2}+c, \quad \forall e \in \mathbb{R}^{n},
$$

for some appropriately chosen constant $c \geqslant 0$. Applying the extended generator (31) to $V_{\text {glob }}$, leads to

$$
\begin{aligned}
L V_{\text {glob }}(e) & =e^{\prime}\left(A+A^{\prime}-\lambda(e)\right) e+b^{\prime} b \\
& \leqslant \sigma_{\max }\left[A+A^{\prime}\right]\|e\|^{2}+b^{\prime} b \\
& =\sigma_{\max }\left[A+A^{\prime}\right]\left(V_{\text {glob }}(e)-c\right)+b^{\prime} b \quad \forall e \in \mathbb{R}^{n},
\end{aligned}
$$

where $\sigma_{\max }\left[A+A^{\prime}\right]$ denotes the largest singular value of $A+A^{\prime}$. Setting $c:=b^{\prime} b / \sigma_{\max }\left[A+A^{\prime}\right]$, we conclude that $L V_{\text {glob }}(e) \leqslant \sigma_{\max }\left[A+A^{\prime}\right] V_{\text {glob }}(e), \forall e \in \mathbb{R}^{n}$ and global existence of solution follows from (i) in Theorem 2, regardless of how we choose $\lambda(e)$.

To establish mean stability, we define

$$
V_{\alpha}(e):=\left(e^{\prime} P e\right)^{\alpha}, \quad \forall e \in \mathbb{R}^{n},
$$

with $\alpha \geqslant 1$. Applying the extended generator (31) to $V_{\alpha}$, we obtain

$$
\begin{gathered}
L V_{\alpha}(e)=\alpha\left(e^{\prime} P e\right)^{\alpha-1} e^{\prime}\left(P A+A^{\prime} P\right) e-\lambda(e)\left(e^{\prime} P e\right)^{\alpha} \\
+\frac{1}{2 \epsilon}\left(\left((e+\sqrt{\epsilon} b)^{\prime} P(e+\sqrt{\epsilon} b)\right)^{\alpha}\right. \\
\left.+\left((e-\sqrt{\epsilon} b)^{\prime} P(e-\sqrt{\epsilon} b)\right)^{\alpha}-2\left(e^{\prime} P e\right)^{\alpha}\right), \quad \forall e \in \mathbb{R}^{n}
\end{gathered}
$$

which can be written as

$$
L V_{\alpha}(e)=-\rho V_{\alpha}(e)+c(e), \quad \forall e \in \mathbb{R}^{n}
$$


for any constant $\rho>0$ and

$$
\begin{aligned}
c(e):=\alpha\left(e^{\prime} P e\right)^{\alpha-1} e^{\prime}\left(P A+A^{\prime} P-\frac{\lambda(e)-\rho}{\alpha} P\right) e \\
+\frac{1}{2 \epsilon}\left(\left((e+\sqrt{\epsilon} b)^{\prime} P(e+\sqrt{\epsilon} b)\right)^{\alpha}\right. \\
\left.+\left((e-\sqrt{\epsilon} b)^{\prime} P(e-\sqrt{\epsilon} b)\right)^{\alpha}-2\left(e^{\prime} P e\right)^{\alpha}\right) .
\end{aligned}
$$

Since $P$ is positive definite, the dominant terms in $c(e)$ as $e \rightarrow \infty$ are the powers in $\left(e^{\prime} P e\right)^{\alpha}$ :

$$
\alpha\left(e^{\prime} P e\right)^{\alpha-1} e^{\prime}\left(P A+A^{\prime} P-\frac{\lambda(e)-\rho}{\alpha}\right) e
$$

which is negative as long as

$$
P A+A^{\prime} P-\frac{\lambda(e)-\rho}{\alpha} P<0 .
$$

When $\lambda(e)$ is radially unbounded, the above inequality holds for sufficient large $e$ and we conclude that

$$
c_{\max }=\sup _{e \in \mathbb{R}^{n}} c(e)<\infty .
$$

In this case,

$$
L V_{\alpha}(e) \leqslant-\rho V_{\alpha}(e)+c_{\max }, \quad \forall e \in \mathbb{R}^{n}
$$

and we can use (iii) in Theorem 3 to conclude that the SDSHS is mean- $V_{\alpha}$ stable and consequently the $2 \alpha$-moment of the error $\mathbb{E}\left[\|e\|^{2 \alpha}\right]$ is bounded, for every $\alpha \geqslant 1$.

When $\lambda(e)$ is bounded:

$$
\exists \kappa>0:\|e\|>\kappa \Rightarrow \lambda(e)=\lambda_{\infty}, \quad \forall e \in \mathbb{R}^{n},
$$

mean- $V_{\alpha}$ stability can be established if there is a positive definite matrix $P$ and a positive constant $\rho$ for which

$$
\begin{aligned}
P A+A^{\prime} P & -\frac{\kappa-\rho}{\alpha} P \\
= & P\left(A-\frac{\kappa-\rho}{2 \alpha} I\right)+\left(A-\frac{\kappa-\rho}{2 \alpha} I\right)^{\prime} P<0
\end{aligned}
$$

which holds if and only if all the eigenvalues of $A$ have real part strictly less than $\frac{\kappa}{2 \alpha}$. When the matrix $A$ has no eigenvalues with positive real part, this condition always holds and the $2 \alpha$-moment of the error $\mathbb{E}\left[\|e\|^{2 \alpha}\right]$ is bounded, for every $\alpha \geqslant 1$. However, when $A$ has any eigenvalue with positive real part, the error $\mathbb{E}\left[\|e\|^{2 \alpha}\right]$ is bounded only for

$$
\alpha<\frac{\kappa}{2 \lambda_{\mathbb{R}}(A)},
$$

where $\lambda_{\mathbb{R}}(A)$ denotes the real part of the eigenvalue of $A$ with largest real part.

\section{STOCHASTIC HYBRID INCLUSIONS}

\section{A. Introduction}

The rest of this tutorial focuses on stochastic hybrid inclusions, which permit non-unique solutions. In contrast to the systems considered in the first part of this tutorial, stochastic hybrid inclusions may include state conditions that force the system's state to jump. The goal of this part of the tutorial is to expose the research community to this class of systems and their utility, to describe the results that have been developed so far, and to indicate the open problems that must be solved to complete the story on stability theory for these systems. Stochastic hybrid inclusions have appeared recently in [2], [3], [4], [5], [6]; the formulation takes inspiration from non-stochastic hybrid inclusions [18], [19], [15], which are discussed briefly below. Closely related results for stochastic difference inclusions, where randomness interacts with non-uniqueness for purely discrete-time systems, have appeared in [20], [21], [22], [23], [24], [25], [26]. Earlier, independent progress has also been made on stochastic differential inclusions; see [27] or [28] for example.

\section{B. Non-stochastic hybrid inclusions}

The model for a non-stochastic hybrid inclusion with state $x \in \mathbb{R}^{n}$ is written formally as

$$
\begin{aligned}
& x \in C, \quad \dot{x} \in F(x) \\
& x \in D, \quad x^{+} \in G(x) .
\end{aligned}
$$

The set $C \subset \mathbb{R}^{n}$, called the flow set, indicates where continuous evolution is allowed. The set $D \subset \mathbb{R}^{n}$, called the jump set, indicates where instantaneous change is allowed. The mappings $F$ and $G$ are set-valued mappings, that is, for each $x \in \mathbb{R}^{n}, F(x)$ and $G(x)$ may be subsets of $\mathbb{R}^{n}$ including the empty set; $F$ is called the flow map, and $G$ is called the jump map. Numerous examples of non-stochastic hybrid inclusions, including specification of the flow and jump sets, and flow and jump maps, can be found in [18], [19], [15]. See also the examples below in Section VII.

Since the model (32) has no preference for flowing behavior over jumping behavior, the time domain of a solution must combine continuous time and discrete time on equal footing. A solution candidate of (32) is a hybrid arc, which is a function $\phi: \operatorname{dom}(\phi) \rightarrow \mathbb{R}^{n}$ such that:

1) $\operatorname{dom}(\phi)$ is a hybrid time domain ${ }^{3}$;

2) for each $i \in \mathbb{Z}_{\geqslant 0}$, if $\operatorname{dom}(\phi) \cap\left(\mathbb{R}_{\geqslant 0} \times\{i\}\right)=: \mathcal{I}_{i} \times\{i\}$ is nonempty then the mapping $\phi(\cdot, i): \mathcal{I}_{i} \rightarrow \mathbb{R}^{n}$ is locally absolutely continuous.

A solution of (32) is a hybrid arc $\phi$ that satisfies:

- $\phi(0,0) \in C \cup D$;

- If $(r, j),(s, j) \in \operatorname{dom}(\phi)$ and $r<s$ then $\phi(t, j) \in C$ and $\frac{d}{d t} \phi(t, j) \in F(\phi(t, j))$ for almost all $t \in[r, s] ;^{4}$

- If $(t, j),(t, j+1) \in \operatorname{dom}(\phi)$ then $\phi(t, j+1) \in G(\phi(t, j))$.

A rich stability theory [15, Ch. 7] has been developed for the systems in (32) under the following mild regularity

\footnotetext{
3 Let $\mathbb{R}_{\geqslant 0}$ denote the nonnegative real numbers and $\mathbb{Z}_{\geqslant 0}$ denote the nonnegative integers. A hybrid time domain is defined in terms of a compact hybrid time domain, the latter being a subset of $\mathbb{R}_{\geqslant 0} \times \mathbb{Z}_{\geqslant 0}$ of the form $\sum_{j=0}^{J}\left[t_{j}, t_{j+1}\right] \times\{j\}$ for real numbers $0=t_{0} \leqslant t_{1} \leqslant \cdots \leqslant t_{J+1}$. A hybrid time domain is a set $E \subset \mathbb{R}_{\geqslant 0} \times \mathbb{Z}_{\geqslant 0}$ such that, for each $(T, J) \in E$, $E \cap([0, T] \times\{0, \ldots, J\})$ is a compact hybrid time domain.

${ }^{4}$ The integral version of this condition is
}

$$
\phi\left(t_{2}, j\right)-\phi\left(t_{1}, j\right)=\int_{t_{1}}^{t_{2}} f(t) d t
$$

for all $t_{1}<t_{2}$ satisfying $r \leqslant t_{1}<t_{2} \leqslant s$ and for some measurable function $f:[r, s] \rightarrow \mathbb{R}^{n}$ that satisfies $f(t) \in F(\phi(t, j))$ for all $t \in[r, s]$. 
conditions, which parallel the conditions in Assumption 1 provided later for stochastic hybrid inclusions and give the system (32) a strong "well-posedness" property [15, Ch. 6]:

1) $C, D \subset \mathbb{R}^{n}$ are closed;

2) $F: \mathbb{R}^{n} \rightrightarrows \mathbb{R}^{n}$ is outer semicontinuous ${ }^{5}$ and locally bounded ${ }^{6}$ with nonempty convex values on $C$;

3) $G: \mathbb{R}^{n} \rightrightarrows \mathbb{R}^{n}$ is outer semicontinuous and locally bounded with nonempty values on $D$.

With this framework as a background, we move to the stochastic case.

\section{Formal model}

Now we consider stochastic hybrid systems with state $x \in$ $\mathbb{R}^{n}$ written formally as

$$
\begin{aligned}
& x \in C, \quad d x \in F(x) d t+B(x) d w \\
& x \in D, \quad x^{+} \in G\left(x, v^{+}\right) \quad v \sim \mu(\cdot) .
\end{aligned}
$$

The set $C \subset \mathbb{R}^{n}$, called the flow set, indicates where continuous evolution is allowed. The set $D \subset \mathbb{R}^{n}$, called the jump set, indicates where instantaneous change is allowed. The mappings $F, B$, and $G$ are set-valued mappings; $F$ is called the drift map, $B$ is called the dispersion map, and $G$ is called the jump map. The symbol $v^{+}$holds the place of a sequence of independent, identically distributed (i.i.d.) random variables $\mathbf{v}_{i}: \Omega \rightarrow \mathbb{R}^{m}, i \in \mathbb{Z}_{\geqslant 1}$, defined on a probability space $(\Omega, \mathcal{F}, \mathbb{P})$, and $\mu$ denotes the associated probability distribution. The symbol $w$ represents a Brownian motion defined on the same probability space.

Spontaneous transitions, like those considered in the first part of this tutorial, can be converted to forced transitions that fit the model (33). This is done by augmenting the state $x$ with a timer variable $\tau \in \mathbb{R}$, yielding a combined state $\left(x^{\top}, \tau\right)^{\top}$. The variable $\tau$ is given continuous-time dynamics $\dot{\tau} \in-\Lambda(x)$, where the values of $\Lambda: \mathbb{R}^{n} \rightrightarrows \mathbb{R}$ are subsets of $\mathbb{R}_{\geqslant 0}$ and are related to the intensities of Section II-C. The new flow set is $C \times \mathbb{R}_{\geqslant 0}$. The jumps of the variable $\tau$ are driven by a sequence of i.i.d. random variables $\widetilde{\mathbf{v}}_{i}: \Omega \rightarrow$ $\mathbb{R}^{m}, i \in \mathbb{Z}_{\geqslant 1}$, defined on the probability space $(\Omega, \mathcal{F}, \mathbb{P})$, often but not necessarily corresponding to an exponential distribution. The jump dynamics for $\tau$ are $\tau^{+}=\tau$ when $(x, \tau) \in D \times \mathbb{R}_{>0}, \tau^{+}=\widetilde{v}^{+}$when $(x, \tau) \in(C \backslash D) \times\{0\}$ and $\tau^{+} \in\left\{\tau, \widetilde{v}^{+}\right\}$when $(x, \tau) \in D \times\{0\}$. The overall jump set is $\left(D \times \mathbb{R}_{\geqslant 0}\right) \cup(C \times\{0\})$.

We acknowledge here that, in the presence of non-unique solutions, this conversion has some (not completely satisfying) consequences when combined with the causality constraints that are a part of the subsequent definition of a solution in Section VIII. On the other hand, for systems with unique solutions, like in the earlier sections, these consequences are benign.

\footnotetext{
${ }^{5}$ That is, for each sequence $\left(x_{i}, y_{i}\right) \rightarrow(x, y)$ satisfying $y_{i} \in F\left(x_{i}\right)$ for all $i \in \mathbb{Z}_{0} \geqslant 0$ we have $y \in F(x)$.

${ }^{6}$ That is, for each $x \in \mathbb{R}^{n}$ there exists an open neighborhood $U$ of $x$ such that $F(U):=\cup_{y \in U} F(y)$ is bounded.
}

\section{EXAMPLES}

\section{A. Sampled-data stochastic control systems}

Consider a sampled-data stochastic control system with state $x_{p} \in \mathbb{R}^{n_{p}}$, input $u_{p} \in \mathbb{R}^{m_{p}}$ and output $y_{p} \in \mathbb{R}^{r_{p}}$ of the form

$$
d x_{p}=f_{p}\left(x_{p}, u_{p}\right) d t+b_{p}\left(x_{p}, u_{p}\right) d w, \quad y_{p}=h_{p}\left(x_{p}, u_{p}\right)
$$

where the signal $y_{p}$ is measured every $T>0$ seconds, at which point the signal $u_{p}$ is updated. The update rule is generated by a control system with state $x_{c} \in \mathbb{R}^{n_{c}}$, input $u_{c} \in \mathbb{R}^{r_{p}}$, and output $y_{c} \in \mathbb{R}^{m_{p}}$ of the form

$$
\dot{x}_{c}=f_{c}\left(x_{c}, u_{c}\right), \quad y_{c}=h_{c}\left(x_{c}, u_{c}\right) .
$$

The measurement and control updates are affected through a timer variable $\tau \in \mathbb{R}$, with continuous evolution allowed when $\tau \in[0, T]$ and jumps allowed when $\tau=T$. The jumps are $u_{p}^{+}=y_{c}$ and $u_{c}^{+}=y_{p}$. Define $n:=n_{p}+m_{p}+n_{c}+r_{p}$, $z:=\left(x_{p}^{\top}, u_{p}^{\top}, x_{c}^{\top}, u_{c}^{\top}\right)^{\top} \in \mathbb{R}^{n}$,

$$
\begin{aligned}
& f(z):=\left[\begin{array}{c}
f\left(x_{p}, u_{p}\right) \\
0 \\
f_{c}\left(x_{c}, u_{c}\right) \\
0
\end{array}\right], b(z):=\left[\begin{array}{c}
b_{p}\left(x_{p}, u_{p}\right) \\
0 \\
0 \\
0
\end{array}\right] \\
& g(z):=\left[\begin{array}{c}
x_{p} \\
h_{c}\left(x_{c}, u_{c}\right) \\
x_{c} \\
h_{p}\left(x_{p}, u_{p}\right)
\end{array}\right] .
\end{aligned}
$$

The overall system is then modeled in the form (33) with state $x:=\left(z^{\top}, \tau\right)^{\top} \in \mathbb{R}^{n+1}$, flow set $\mathbb{R}^{n} \times[0, T]$, jump set $\mathbb{R}^{n} \times\{T\}$, drift and dispersion maps

$$
F(x):=\left[\begin{array}{c}
f(z) \\
1
\end{array}\right], \quad B(x):=\left[\begin{array}{c}
b(z) \\
0
\end{array}\right],
$$

and jump map

$$
G(x):=\left[\begin{array}{c}
g(z) \\
0
\end{array}\right] .
$$

At times it may be reasonable to consider the situation where the mapping $h_{c}$, and thus $G$, is discontinuous. In turn, like in the non-stochastic case [15, Chapters 6 \& 7], assessing robustness of stability motivates replacing the discontinuous function $G$ by its outer semicontinuous hull $\bar{G}[16$, p. 154 155], which is the set-valued mapping whose graph matches the closure of the graph of $G$, i.e.,

$$
\begin{aligned}
\left\{(y, z) \in \mathbb{R}^{n+1} \times \mathbb{R}^{n+1}: y \in \bar{G}(z)\right\} \\
=\overline{\left\{(y, z) \in \mathbb{R}^{n+1} \times \mathbb{R}^{n+1}: y=G(z)\right\} .}
\end{aligned}
$$

In this way, non-unique solutions are produced and correspond to the solutions that might arise when arbitrarily small perturbations are added to $z$, especially to $x_{c}$ or $u_{c}$, in the process of evaluating $G$. 
B. Average dwell-time switching among stochastic differential equations

As observed in [29] or [30], an automaton can be constructed to enforce an "average dwell-time" constraint [31] on the frequency of mode switching, and to simultaneously capture all switching signals that satisfy the average dwelltime constraint. In particular, the class of switching signals where the number of switches in an interval of length $\ell$ is bounded by $\delta \ell+N$, where $\delta \in \mathbb{R}_{\geqslant 0}$ and $N \in \mathbb{Z}_{\geqslant 1}$, is generated by the hybrid automaton

$$
\begin{array}{ll}
\tau \in[0, N] & \dot{\tau} \in[0, \delta] \\
\tau \in[1, N] & \tau^{+}=\tau-1 .
\end{array}
$$

Now consider a switching stochastic differential equation

$$
d \xi=f_{q}(\xi) d t+b_{q}(\xi) d w \quad q \in \mathcal{Q}
$$

where $\mathcal{Q} \subset \mathbb{R}$ is a set with a finite number of elements. Combining (36) and (37) and making the switching variable $q$ a state yields a system of the form (33) with state $x:=$ $\left(\xi^{\top}, q, \tau\right)^{\top}$, flow set $C:=\mathbb{R}^{n} \times \mathcal{Q} \times[0, N]$, jump set $D:=$ $\mathbb{R}^{n} \times \mathcal{Q} \times[1, N]$, drift and dispersion maps

$$
F(x):=\left[\begin{array}{c}
f_{q}(\xi) \\
0 \\
{[0, \delta]}
\end{array}\right], \quad B(x):=\left[\begin{array}{c}
b_{q}(\xi) \\
0 \\
0
\end{array}\right]
$$

and jump map

$$
G(x):=\left[\begin{array}{c}
\xi \\
\mathcal{Q}_{q} \\
\tau-1
\end{array}\right]
$$

where $\mathcal{Q}_{q} \subset \mathcal{Q} \backslash\{q\}$ denotes the set of admissible next values of the mode when the current value is $q$. Taking $\mathcal{Q}_{q}=\mathcal{Q} \backslash\{q\}$ for all $q \in \mathcal{Q}$ corresponds to the situation where there is no restriction on the next mode.

This model allows for all (stochastic) switching signals whose sample paths satisfy the given average dwell-time switching constraint and that depend causally on the Brownian motion $w$. The causality constraint will become clearer when the solution concept is described in Section VIII. Non-uniqueness arises since there are many such switching signals. This behavior is manifest in the substantial overlap of the sets $C$ and $D$, together with the set-valued flow map and jump map.

It is trivial to also consider a model where $\xi$ jumps, perhaps randomly, when $q$ jumps by replacing the $\xi$-component of the mapping $G$ in (39) with a jump map $\widetilde{G}_{q}\left(\xi, v^{+}\right)$where $v^{+}$is a placeholder for the elements of a random process.

\section{A stochastic bouncing ball system}

Consider a simplified bouncing ball model where the coefficient of restitution at impacts is random. Let $x_{1} \in \mathbb{R}$ denote the height of the ball above the ground and let $x_{2} \in \mathbb{R}$ denote the ball's velocity. We define the flow set $C:=\mathbb{R}_{\geqslant 0} \times \mathbb{R}$ and the jump set $D:=\{0\} \times \mathbb{R}_{\leqslant 0}$. The flow map can be taken to be $F(x):=\left(x_{2},-\gamma\right)^{\top}$ for $x \neq 0$, where $\gamma>0$ is the force due to gravity, and $F(0):=(0,[-\gamma, 0])^{\top}$.
The definition of $F$ at the origin induces a solution at the origin that flows, without moving from the origin, for all time. We consider a jump map of the form $G(x, v):=-v x$ where $v>0$ is the random coefficient of restitution. Due to the structure of $G$, there is also a solution from the origin that "jumps", without moving from the origin. There are also solutions from the origin that combine flowing and jumping, in arbitrary fashion, without moving from the origin.

\section{The nefarious nurse}

Building on the academic, nefarious nurse example in [2], consider a system with state $x \in \mathbb{R}^{3}$, flow set $C:=\mathbb{S}^{1} \times \mathbb{R}_{\geqslant 0}$, and jump set $\left\{x \in \mathbb{S}^{1}: x_{1}=-x_{2}\right\} \times \mathbb{R}_{\geqslant 0}$. The drift map $F$ and dispersion map $B$ are taken to be

$$
F(x):=\left[\begin{array}{c}
x_{2} \\
-x_{1} \\
-\varepsilon x_{3}+c
\end{array}\right], \quad B(x):=\left[\begin{array}{c}
0 \\
0 \\
\tanh \left(x_{3}\right)
\end{array}\right]
$$

where $c \geqslant 0$ and $0<\varepsilon \ll 1$. The jump map $G$ is taken to be

$$
G(x, v):=\left[\begin{array}{c}
0 \\
\operatorname{sgn}\left(x_{2}\right) \\
\sigma_{\operatorname{sgn}\left(x_{2}\right)}(v) x_{3}
\end{array}\right], \quad v \sim \mu(\cdot)
$$

where $\mu(\{1\})=\mu(\{-1\})=0.5$, and $\sigma_{-1}, \sigma_{1}:\{-1,1\} \rightarrow \mathbb{R}$ are such that $\sigma_{g}:=\sigma_{1}(1)=\sigma_{-1}(-1) \in(0,1)$ and $\sigma_{b}:=\sigma_{1}(-1)=\sigma_{-1}(1) \in(1, \infty)$. Non-unique solutions are enabled by the fact that $D$ is a subset of $C$ and it is possible to flow through $D$ without jumping. As will be discussed subsequently, solutions are required to depend causally on the random inputs to the system, including the inputs $\mathbf{v}_{i}: \Omega \rightarrow\{-1,1\}$. It follows from the structure of the functions $\sigma_{i}$ that, for $\varepsilon>0$ sufficiently small, it is possible to select jumps non-causally so that an increase in the size of $x_{3}$ occurs at every jump and so that at least one jump occurs each time $\left(x_{1}, x_{2}\right)$ rotates around the circle. On the other hand, for certain values of $\sigma_{i}(j)$, causal solutions tend to drift toward the origin. This behavior will be explained later when considered Lyapunov-based sufficient conditions for asymptotic stability and recurrence.

\section{SOLUTIONS}

\section{A. Construction}

The solution concept for the stochastic system (33) borrows heavily from the solution concept for non-stochastic hybrid systems found in [32] or [18], [19], [15]. In the non-stochastic case, a solution candidate is a hybrid arc. In the stochastic case, a sample path of a solution candidate is a relaxed hybrid arc. A relaxed hybrid arc is a function $\phi: \operatorname{dom}(\phi) \rightarrow \mathbb{R}^{n}$ such that:

1) $\operatorname{dom}(\phi)$ is a hybrid time domain

2) viewed as a set-valued mapping from $\mathbb{R}^{2}$ to $\mathbb{R}^{n}, \phi$ is outer semicontinuous; that is, for each sequence $\left(t_{i}, j_{i}, z_{i}\right) \rightarrow(t, j, z)$ satisfying $z_{i}=\phi\left(t_{i}, j_{i}\right)$ for all $i \in \mathbb{Z}_{\geqslant 0}$ we have $z=\phi(t, j)$.

\footnotetext{
${ }^{7}$ See Footnote 3.
} 
Item 2 is equivalent to the condition that the $\operatorname{set} \operatorname{graph}(\phi):=$ $\left\{(t, j, z) \in \mathbb{R}^{n+2}: z=\phi(t, j)\right\}$ is closed; see [16, Thm. 5.7(a)]. It also implies that for each $i \in \mathbb{Z}_{\geqslant 0}$, if $\operatorname{dom}(\phi) \cap$ $\left(\mathbb{R}_{\geqslant 0} \times\{i\}\right)=: \mathcal{I}_{i} \times\{i\}$ is nonempty then the mapping $\phi(\cdot, i): \mathcal{I}_{i} \rightarrow \mathbb{R}^{n}$ is continuous. In contrast, a hybrid arc is such that this function is locally absolutely continuous.

A solution candidate for (33) is a mapping $\mathbf{x}$ defined on the probability space $(\Omega, \mathcal{F}, \mathbb{P})$ with the property that, for almost every $\omega \in \Omega, \mathbf{x}(\omega)$ is a relaxed hybrid arc. ${ }^{8}$ In characterizing what is meant by a solution, we will use the mappings $\mathbf{T}_{\dashv}: \Omega \rightarrow\left(\mathbb{R}_{\geqslant 0} \cup\{\infty\}\right)$ and $\mathbf{J}_{\dashv}: \Omega \rightarrow$ $\left(\mathbb{Z}_{\geqslant 0} \cup\{\infty\}\right)$ defined by:

$$
\left(\mathbf{T}_{\dashv}(\omega), \mathbf{J}_{\dashv}(\omega)\right):=\sup \{(t, j) \in \operatorname{dom}(\mathbf{x}(\omega))\} .
$$

For the sake of a comprehensive and natural stability theory for (33), a solution candidate for (33) should have an appropriate causal dependence on the stochastic processes that drive the system. As spelled out in [5], this causal structure can be expressed in terms of a hybrid filtration $\left\{\mathcal{F}_{t, j}\right\}_{(t, j) \in \mathbb{R}_{\geqslant 0} \times \mathbb{Z}_{\geqslant 0}}$ of the underlying probability space, generated from the Brownian motion $w$ and the random process $\left\{\mathbf{v}_{i}\right\}_{i=1}^{\infty}$, that is right-continuous in $t$. We refer the reader to [5, §III] for more details. A solution candidate for (33) is an adapted solution candidate if, for each $(t, j) \in \mathbb{R}_{\geqslant 0} \times \mathbb{Z}_{\geqslant 0}$, the set-valued mapping

$$
\omega \mapsto \operatorname{graph}(\mathbf{x}(\omega)) \cap([0, t] \times\{0, \ldots, j\})
$$

is $\mathcal{F}_{t, j}$-measurable ${ }^{9}$ and

$$
\left.\left\{\omega \in \Omega: \mathbf{T}_{\dashv}(\omega) \leqslant t, \mathbf{J}_{\dashv}(\omega)\right) \leqslant j\right\} \in \mathcal{F}_{t, j} .
$$

An adapted solution candidate is a solution if its sample paths satisfy the constraints associated with (33) almost surely, in a manner similar to the way solutions of (32) satisfy the constraints in (32) as described near the end of Section VI$\mathrm{B}$, using a stochastic integral formulation of the continuoustime constraints. For more details, we refer the reader to [5, $\oint \mathrm{IV}] .{ }^{10}$ We use $\mathcal{S}_{\mathbf{r}}(K)$ to denote the set of solutions to (33) with initial conditions belonging to $K \subset \mathbb{R}^{n}$.

\section{B. Hybrid stopping times}

A pair of $\mathcal{F}$-measurable mappings $\mathbf{T}: \Omega \rightarrow\left(\mathbb{R}_{\geqslant 0} \cup\{\infty\}\right)$ and $\mathbf{J}: \Omega \rightarrow\left(\mathbb{Z}_{\geqslant 0} \cup\{\infty\}\right)$ is called a hybrid stopping time if, for all $(t, j) \in \mathbb{R}_{\geqslant 0} \times \mathbb{Z}_{\geqslant 0}$,

$$
\{\omega \in \Omega:(\mathbf{T}(\omega), \mathbf{J}(\omega)) \leq(t, j)\} \in \mathcal{F}_{t, j}
$$

\footnotetext{
${ }^{8}$ As such, due to Item 2 above, $\mathbf{x}$ can be thought of as a mapping from $\Omega$ to the space of (not identically empty) outer semicontinuous set-valued mappings from $\mathbb{R}^{2}$ to $\mathbb{R}^{n}$. According to [16, Theorem 5.50], this space equipped with the graph distance metric, is a separable, complete, locally compact metric space.

${ }^{9}$ Measurability of a set-valued mapping with values that are subsets of a Euclidean space is discussed in great detail in [16, Ch. 14]. A set-valued mapping $M: \Omega \rightrightarrows \mathbb{R}^{n}$ is $\mathcal{F}_{t, j}$-measurable if, for each open set $\mathcal{O}$, $M^{-1}(\mathcal{O}):=\{\omega \in \Omega: M(\omega) \cap \mathcal{O} \neq \varnothing\} \in \mathcal{F}_{t, j}$.

${ }^{10}$ Effectively, the solution concept in [5] focuses on the notion of strong solutions to the constrained stochastic differential inclusion (33a) whereas a weaker solution concept is sometimes used for stochastic differential inclusions; see [27] or [28]
}

where $(\mathbf{T}(\omega), \mathbf{J}(\omega)) \leq(t, j)$ means $\mathbf{T}(\omega) \leqslant t$ and $\mathbf{J}(\omega) \leqslant j$. Note that the condition (43) implies that the time $\left(\mathbf{T}_{\dashv}, \mathbf{J}_{\dashv}\right)$ defined in (42) is a hybrid stopping time. It turns out, as stated in [5, Lemma 2], that if two hybrid stopping times $\left(\mathbf{T}_{1}, \mathbf{J}_{1}\right)$ and $\left(\mathbf{T}_{2}, \mathbf{J}_{2}\right)$ satisfy

$$
\begin{aligned}
& \left(\mathbf{T}_{1}(\omega), \mathbf{J}_{1}(\omega)\right) \leq\left(\mathbf{T}_{2}(\omega), \mathbf{J}_{2}(\omega)\right) \text { or } \\
& \left(\mathbf{T}_{2}(\omega), \mathbf{J}_{2}(\omega)\right) \leq\left(\mathbf{T}_{1}(\omega), \mathbf{J}_{1}(\omega)\right)
\end{aligned}
$$

for almost all $\omega \in \Omega$ then $\left(\mathbf{T}_{1} \vee \mathbf{T}_{2}, \mathbf{J}_{1} \vee \mathbf{J}_{2}\right)$ and $\left(\mathbf{T}_{1} \wedge\right.$ $\left.\mathbf{T}_{2}, \mathbf{J}_{1} \wedge \mathbf{J}_{2}\right)$ are hybrid stopping times, where $a \vee b:=$ $\max \{a, b\}$ and $a \wedge b:=\min \{a, b\}$. The condition (44) will hold when the two stopping times $\left(\mathbf{T}_{1}, \mathbf{J}_{1}\right)$ and $\left(\mathbf{T}_{2}, \mathbf{J}_{2}\right)$ are generated from the same hybrid time domain almost surely, which is the case if they are generated from a particular solution x. As indicated in [5, Lemma 5], examples of such hybrid stopping times generated from $\mathbf{x}$ include the first exit time from a closed set $\mathcal{C}$ and the smallest time greater than $\tau \geqslant 0$, i.e.,

$$
\begin{aligned}
& \left(\mathbf{T}_{\mathcal{C}}(\omega), \mathbf{J}_{\mathcal{C}}(\omega)\right):=\inf \left\{(t, j) \in \operatorname{dom}(\mathbf{x}(\omega)): \mathbf{x}_{\omega}(t, j) \notin \mathcal{C}\right\} \\
& \left(\mathbf{T}_{\tau}(\omega), \mathbf{J}_{\tau}(\omega)\right):=\inf \{(t, j) \in \operatorname{dom}(\mathbf{x}(\omega)): t+j>\tau\}
\end{aligned}
$$

with the understanding that the infimum over the empty set is infinity.

Given a solution $\mathbf{x}$ and a hybrid stopping time $(\mathbf{T}, \mathbf{J})$ we call the latter a hybrid stopping time of $\mathbf{x}$, and write $(\mathbf{T}, \mathbf{J}) \in$ $\operatorname{hst}(\mathbf{x})$, if $(\mathbf{T}(\omega), \mathbf{J}(\omega)) \in \operatorname{dom}(\mathbf{x}(\omega))$ for almost all $\omega \in \Omega$. We note that, for each $\mathbf{x} \in \mathcal{S}_{\mathbf{r}}\left(\mathbb{R}^{n}\right), \tau \geqslant 0$, and compact $\mathcal{C} \subset \mathbb{R}^{n}$, the hybrid stopping time

$$
\left(\mathbf{T}_{\dashv} \wedge \mathbf{T}_{\tau} \wedge \mathbf{T}_{\mathcal{C}}, \mathbf{J}_{\dashv} \wedge \mathbf{J}_{\tau} \wedge \mathbf{J}_{\mathcal{C}}\right)
$$

is a hybrid stopping time of $\mathbf{x}$; moreover, in the absence of finite escape times almost surely, the hybrid stopping time

$$
\left(\mathbf{T}_{\dashv} \wedge \mathbf{T}_{\tau}, \mathbf{J}_{\dashv} \wedge \mathbf{J}_{\tau}\right)
$$

is a hybrid stopping time of $\mathbf{x}$.

For later use, we make the following definitions.

- Given a solution $\mathbf{x}$, for each $i \in \mathbb{Z}_{\geqslant 0}$ we define the mappings $\mathbf{T}_{i}: \Omega \rightarrow[0, \infty]$ by

$$
\mathbf{T}_{i}(\omega):=\inf \left\{s \in \mathbb{R}_{\geqslant 0}:(s, i) \in \operatorname{dom}(\mathbf{x}(\omega))\right\}
$$

where the infimum over the empty set is taken to be $\infty$. It turns out [5, Lemma 3] that, for each $i \in \mathbb{Z}_{\geqslant 0},\left(\mathbf{T}_{i}, i\right)$ and $\left(\mathbf{T}_{i+1}, i\right)$ are hybrid stopping times.

- Given a solution $\mathbf{x}$ and a hybrid stopping time $(\mathbf{T}, \mathbf{J})$ of $\mathbf{x}$, for each $\omega \in \Omega$ and $s \in[0, \mathbf{T}(\omega)]$ we define $\mathbf{J}_{s}(\omega):=\sup \left\{j \in \mathbb{Z}_{\geqslant 0}:(s, j) \in \operatorname{dom}(\mathbf{x}(\omega))\right\}$.

\section{Stability THEORY}

\section{A. Preamble}

We consider several different stability notions. Many of these properties are similar in spirit to the definitions that were given in Section IV-A, and are also summarized in the survey on stability theory for stochastic hybrid systems in [7]. First, as emphasized in [19, p. 58], we reiterate that a 
widely applicable stability theory for hybrid systems must transcend the study of stability of equilibria. This assertion becomes evident when considering many of the examples in Section VII. Indeed, it is clear that timer variables associated with switching do not converge to a point, and switching signals do not typically converge to a point. Instead, these variables evolve in a closed set, typically one that is compact. Similarly, the rotational clock variables in the nefarious nurse example do not converge, but evolve in a compact set, namely the unit circle. Consequently, when studying asymptotic stability, we consider this property for closed or compact sets. For technical reasons, in the presence of stochastic differential inclusions, it is easier to given sufficient conditions for asymptotic stability (in probability) of compact sets rather than more general closed sets.

In addition to asymptotic stability, we also discuss a property called recurrence, which is usually applied to an open set. Recurrence of an open set $\mathcal{O}$ captures the property that solutions reach $\mathcal{O}$ (almost surely) from any initial condition. Then, by semi-group properties, it is reached recurrently. There are several reasons to consider recurrence. First, it may give additional sharper information about the behavior of solutions if, for example, within a small neighborhood of a compact asymptotically stable set there is another smaller open set that is recurrent. Also, for stochastic systems, it is possible for an open bounded set to be recurrent without there existing a compact, asymptotically stable set. (The simplest example of such a system is the one-dimensional discrete-time system $x^{+}=v^{+}, v \sim \mu(\cdot)$, where $\mu(\cdot)$ corresponds to a Gaussian distribution.) Recurrence of open, bounded sets for non-stochastic hybrid systems has been studied recently in [33], where an equivalent Lyapunov characterization is given.

Finally, we also consider various definitions of stability expressed in terms of the expected value of a function of the distance of the state to a closed or compact set.

Like in Section IV-A, we focus on global stability properties. Local stability properties can also be considered, using ideas like in [34, §8.4.1].

\section{B. Stability definitions}

1) Notation: Here and in what follows, $\alpha \in \mathcal{G}_{\infty}$ if $\alpha$ : $\mathbb{R}_{\geqslant 0} \rightarrow \mathbb{R}_{\geqslant 0}$ is continuous, nondecreasing and unbounded, while $\alpha \in \mathcal{K}_{\infty}$ if $\alpha$ belongs to $\mathcal{G}_{\infty}$, is zero at zero, and is strictly increasing. For a closed set $\mathcal{A} \subset \mathbb{R}^{n}$ and $x \in \mathbb{R}^{n}$, we use the definition $|x|_{\mathcal{A}}:=\inf _{y \in \mathcal{A}}|x-y|$. The symbol $\mathbb{B}$ denotes the closed unit ball and $\mathbb{B}^{\circ}$ denotes the open unit ball. For $\varepsilon>0, \mathcal{A}+\varepsilon \mathbb{B}=\left\{x \in \mathbb{R}^{n}:|x|_{\mathcal{A}} \leqslant \varepsilon\right\}$. Recall that, given a relaxed hybrid arc $\phi: \operatorname{dom}(\phi) \rightarrow \mathbb{R}^{n}, \operatorname{graph}(\phi):=$ $\left\{(t, j, z) \in \mathbb{R}^{n+2}:(t, j) \in \operatorname{dom}(\phi), z=\phi(t, j)\right\}$. We also use of the definitions

$$
\begin{aligned}
& \Gamma_{\geqslant \tau}:=\left\{(s, t) \in \mathbb{R}^{2}: s+t \geqslant \tau\right\}, \\
& \Gamma_{\leqslant \tau}:=\left\{(s, t) \in \mathbb{R}^{2}: s+t \leqslant \tau\right\}, \\
& \Gamma_{<\tau}:=\left\{(s, t) \in \mathbb{R}^{2}: s+t<\tau\right\},
\end{aligned}
$$

and

$$
\begin{aligned}
& \operatorname{graph}_{\geqslant \tau}(\phi):=\operatorname{graph}(\phi) \cap\left(\Gamma_{\geqslant \tau} \times \mathbb{R}^{n}\right), \\
& \operatorname{graph}_{\leqslant \tau}(\phi):=\operatorname{graph}(\phi) \cap\left(\Gamma_{\leqslant \tau} \times \mathbb{R}^{n}\right), \\
& \operatorname{graph}_{<\tau}(\phi):=\operatorname{graph}(\phi) \cap\left(\Gamma_{<\tau} \times \mathbb{R}^{n}\right) .
\end{aligned}
$$

2) Asymptotic stability in probability: We consider uniform global asymptotic stability in probability for a closed set $\mathcal{A} \subset \mathbb{R}^{n}$. These definitions, inspired by similar definitions for classical stochastic systems, have appeared previously in [2] and [5]. In contrast to the definitions given earlier, we do not assume as a part of the stability definition that that the sample paths of a maximal solution have unbounded time domains, let alone that the time domains are unbounded in the direction of ordinary time. Uniform global asymptotic stability in probability is expressed in terms of three subproperties: Lyapunov stability in probability, Lagrange stability in probability, and uniform global attractivity. Each of these properties is expressed in terms of the probability that the graphs of solutions have appropriate properties.

The closed set $\mathcal{A} \subset \mathbb{R}^{n}$ is said to be uniformly Lyapunov stable in probability for (33) if there exists $\mu>0$ such that, for each solution of

$$
x \in C \cap(\mathcal{A}+\mu \mathbb{B}) \quad d x \in F(x) d t+B(x) d w,
$$

there are no finite escape times almost surely and for each $\varepsilon>0$ and $\rho>0$ there exists $\delta>0$ such that

$$
\begin{aligned}
& \mathbf{x} \in \mathcal{S}_{\mathbf{r}}(\mathcal{A}+\delta \mathbb{B}) \Longrightarrow \\
& \mathbb{P}\left(\operatorname{graph}(\mathbf{x}) \subset \mathbb{R}^{2} \times(\mathcal{A}+\varepsilon \mathbb{B})\right) \geqslant 1-\rho .
\end{aligned}
$$

The graph of a sample path $\mathbf{x}(\omega)=: \mathbf{x}_{\omega}$ being a subset of $\mathbb{R}^{2} \times(\mathcal{A}+\varepsilon \mathbb{B})$ means that $\left|\mathbf{x}_{\omega}(t, j)\right|_{\mathcal{A}} \leqslant \varepsilon$ for all $(t, j) \in \operatorname{dom}\left(\mathbf{x}_{\omega}\right)$. Therefore, in words, the condition (47) says that no matter how close we want the solutions to remain to $\mathcal{A}$ (within a distance $\varepsilon>0$ ) with probability no matter how close to one $(1-\rho, \rho>0)$, this property is achieved by starting the solutions sufficiently close to $\mathcal{A}$ (within a distance $\delta>0$ ). This property thus parallels the definition of Lyapunov stability in probability given in Section IV-A and characterized by (15).

The closed set $\mathcal{A} \subset \mathbb{R}^{n}$ is said to be uniformly Lagrange stable in probability for (33) if, for each solution of (33a) there are no finite escape times almost surely and for each $\delta>0$ and $\rho>0$ there exists $\varepsilon>0$ such that (47) holds. In words, this conditions says that no matter how far the solutions start from $\mathcal{A}$ (within a distance $\delta>0$ ) we can find a bound on the worst-case distance from $\mathcal{A}$ (within a distance $\varepsilon>0)$ with probability arbitrarily close to one $(1-\rho, \rho>0)$.

The closed set $\mathcal{A} \subset \mathbb{R}^{n}$ is said to be uniformly globally stable in probability for (33) if it is both uniformly Lyapunov stable in probability and uniformly Lagrange stable in probability.

The closed set $\mathcal{A} \subset \mathbb{R}^{n}$ is said to be uniformly globally attractive in probability (UGAp) for (33) if for each solution of (33a) there are no finite escape times almost surely and 
for each $\Delta>0, \varepsilon>0$, and $\rho>0$ there exists $\tau>0$ such that

$$
\begin{aligned}
& \mathrm{x} \in \mathcal{S}_{\mathbf{r}}(\mathcal{A}+\Delta \mathbb{B}) \Longrightarrow \\
& \mathbb{P}\left(\operatorname{graph}_{\geqslant \tau}(\mathrm{x}) \subset \mathbb{R}^{2} \times\left(\mathcal{A}+\varepsilon \mathbb{B}^{\circ}\right)\right) \geqslant 1-\rho .
\end{aligned}
$$

The graph of a sample path $\mathbf{x}(\omega)=: \mathbf{x}_{\omega}$, restricted to hybrid times greater than or equal to $\tau$, being a subset of $\mathbb{R}^{2} \times\left(\mathcal{A}+\varepsilon \mathbb{B}^{\circ}\right)$ means that $\left|\mathbf{x}_{\omega}(t, j)\right|_{\mathcal{A}}<\varepsilon$ for all $(t, j) \in \operatorname{dom}\left(\mathbf{x}_{\omega}\right)$ satisfying $t+j \geqslant \tau$. Therefore, in words, this condition (48) says that no matter how far from $\mathcal{A}$ the solutions start (within a distance $\Delta>0$ ) and no matter how close to $\mathcal{A}$ we would like the solutions to ultimately be (within a distance $\varepsilon>0$ ) with probability arbitrarily close to one $(1-\rho, \rho>0)$, there is some amount of hybrid time $(\tau>0)$ we can wait to have this level of confidence that the solution has either stopped before this time or else is now constrained to the small neighborhood of $\mathcal{A}$. The waiting time can be taken to be uniform over all solutions starting within a distance $\Delta$ of the set $\mathcal{A}$.

The closed set $\mathcal{A} \subset \mathbb{R}^{n}$ is said to be uniformly globally asymptotically stable in probability (UGASp) for (33) if it is uniformly globally stable in probability and uniformly globally attractive in probability. Based on the discussion in the previous paragraph, this property is similar to, but in some ways stronger than, the definition of asymptotic stability in probability given in Section IV-A.

When we study recurrence, we will find that the following additional Lagrange stability definition is useful. The closure of an open set $\mathcal{O} \subset \mathbb{R}^{n}$ is said to be uniformly Lagrange stable in probability conditioned on stopping at $\mathcal{O}$ if $\overline{\mathcal{O}}$ is uniformly Lagrange stable in probability for the system with data $\left(C \cap \mathcal{O}^{c},(F, B), D \cap \mathcal{O}^{c}, G, \mu\right)$ where $\mathcal{O}^{c}:=\mathbb{R}^{n} \backslash \mathcal{O}$. Intersecting the flow and jump sets with $\mathcal{O}^{c}$ forces the solutions to stop after they jump to $\mathcal{O}$ or when they reach the boundary of $\mathcal{O}$ and attempt to flow into $\mathcal{O}$. This "stopping" procedure partially motivates not insisting that each sample path has an unbounded hybrid time domain; see also [15, Ex. 3.8].

3) Recurrence: We now define uniform global recurrence in probability for an open set. Like in the case of asymptotic stability in probability, the recurrence property is expressed in terms of the graphs of solutions and does not insist that the sample paths have unbounded time domains. Recurrence plays a prominent role in stability theory for stochastic systems. It is the dominant stability property considered in [35] and it is studied extensively in [13, Ch. 3].

The open set $\mathcal{O} \subset \mathbb{R}^{n}$ is said to be uniformly globally recurrent in probability (UGRp) if for each solution of (33a) there are no finite escape times almost surely and for each $\Delta>0$ and $\rho>0$ there exists $\tau>0$ such that

$$
\mathbf{x} \in \mathcal{S}_{\mathbf{r}}(\overline{\mathcal{O}}+\Delta \mathbb{B}) \quad \Longrightarrow \quad \mathbb{P}\left(\Omega_{a, \tau} \cup \Omega_{b, \tau}\right) \geqslant 1-\rho
$$

where

$$
\begin{aligned}
& \Omega_{a, \tau}:=\left\{\omega \in \Omega: \operatorname{graph}_{<\tau}(\mathbf{x}(\omega))=\operatorname{graph}(\mathbf{x}(\omega))\right\} \\
& \Omega_{b, \tau}:=\left\{\omega \in \Omega: \operatorname{graph}_{\leqslant \tau}(\mathbf{x}(\omega)) \cap\left(\mathbb{R}^{2} \times \mathcal{O}\right) \neq \varnothing\right\} .
\end{aligned}
$$

In words, this definition says that no matter how far the solutions start from the closure of $\mathcal{O}$ (within a distance $\Delta>$ $0)$ and no matter how close to one $(1-\rho, \rho>0)$ we want the probability that the solution has reached $\mathcal{O}$, there is some amount of hybrid time $\tau>0$ we can wait to have this level of confidence that the solution has either stopped before this time or else has reached $\mathcal{O}$. The waiting time can be taken to be uniform over all solutions starting within a distance $\Delta$ of the closure of the set $\mathcal{O}$.

It should be clear from the definitions that if a closed set $\mathcal{A} \subset \mathbb{R}^{n}$ is uniformly globally attractive in probability then, for each $\varepsilon>0$, the open set $\mathcal{A}+\varepsilon \mathbb{B}^{\circ}$ is uniformly globally recurrent in probability. Conversely, if the closed set $\mathcal{A} \subset \mathbb{R}^{n}$ is uniformly globally stable in probability and for each pair of strictly positive real numbers $(\delta, \Delta)$ the open set $\left\{x \in \mathbb{R}^{n}:|x|_{\mathcal{A}} \in[0, \delta) \cup(\Delta, \infty)\right\}$ is uniformly globally recurrent in probability then $\mathcal{A}$ is uniformly globally attractive in probability. See [2, Prop. 2.2] and [5, Thm. 1]; cf. [36, p. 167, Thm. 4] and [13, p. 189, Lemma 7.6; p. 261, Thm. 9.6].

Sometimes it is also useful to know (see [2, Prop. 2.4] and [5, Thm. 2]) that an open set $\mathcal{O} \subset \mathbb{R}^{n}$ is uniformly globally recurrent in probability if its closure is uniformly Lagrange stable in probability conditioned on stopping at $\mathcal{O}$ and, for each $\Delta>0$, the set $\mathcal{O} \cup\left(\mathbb{R}^{n} \backslash \overline{\mathcal{O}}+\Delta \mathbb{B}\right)$ is uniformly globally recurrent in probability. Indeed, it may be easier to establish recurrence of the larger open set $\mathcal{O} \cup\left(\mathbb{R}^{n} \backslash \overline{\mathcal{O}}+\Delta \mathbb{B}\right)$. For example, see Section X-E below.

4) Asymptotic stability in expected value: Finally, we turn our attention to asymptotic stability in expected value for a closed set $\mathcal{A} \subset \mathbb{R}^{n}$. There are a variety of possible definitions of asymptotic stability in expected value. For simplicity, we focus only on the properties discussed below, which resemble some of the definitions in Section IV-A with $W(x)=\alpha_{1}\left(|x|_{\mathcal{A}}\right)$ for some $\alpha_{1} \in \mathcal{K}_{\infty}$.

The closed set $\mathcal{A} \subset \mathbb{R}^{n}$ is said to be uniformly Lyapunov stable in expected value for (33) if there exists $\mu>0$ such that, for each solution of

$$
x \in C \cap(\mathcal{A}+\mu \mathbb{B}) \quad d x \in F(x) d t+B(x) d w,
$$

there are no finite escape times almost surely and there exists $\alpha \in \mathcal{K}_{\infty}$ such that, for each $\varepsilon>0$ there exists $\delta>0$ such that

$$
\left.\begin{array}{r}
\mathbf{x} \in \mathcal{S}_{\mathbf{r}}(\mathcal{A}+\delta \mathbb{B}) \\
(\mathbf{T}, \mathbf{J}) \in \operatorname{hst}(\mathbf{x})
\end{array}\right\} \Longrightarrow \mathbb{E}\left[\alpha\left(|\mathbf{x}(\mathbf{T}, \mathbf{J})|_{\mathcal{A}}\right)\right] \leqslant \varepsilon .
$$

In words, the definition says that no matter how small $(\varepsilon>0)$ we desire the expected value of an appropriate $\mathcal{K}_{\infty}$-function of the solution's distance of the state to $\mathcal{A}$ to be, this bound can be guaranteed by starting the solution sufficiently close (within the distance $\delta>0$ ) to the set $\mathcal{A}$. We do not fix the evaluation time to be constant since sample paths do not typically have the same hybrid time domain; hence, it is unlikely that a constant time will belong to almost every time domain of the solution.

The closed set $\mathcal{A} \subset \mathbb{R}^{n}$ is said to be uniformly Lagrange stable in expected value for (33) if, for each solution of (33a) 
there are no finite escape times almost surely and there exists $\alpha \in \mathcal{K}_{\infty}$ such that for each $\delta>0$ there exists $\varepsilon>0$ such that (49) holds. In words, this conditions says that no matter how far the solutions start from $\mathcal{A}$ (within a distance $\delta>0$ ) we can find a bound on the expected value of a $\mathcal{K}_{\infty}$-function of the solution's distance from $\mathcal{A}$ (within a distance $\varepsilon>0$ ).

The closed set $\mathcal{A} \subset \mathbb{R}^{n}$ is said to be uniformly globally stable in expected value for (33) if it is both uniformly Lyapunov stable in expected value and uniformly Lagrange stable expected value for (33).

As mentioned above, there are many different possible definitions of asymptotic stability that are expressed in terms of expected values. Since not all sample paths have the same hybrid time domain, so that hybrid times are also random variables, it is reasonable to include in the value for which the expected value is formed a growing function of the elapsed time. One way to do so is as follows.

The closed set $\mathcal{A} \subset \mathbb{R}^{n}$ is said to be uniformly globally quasi-exponentially stable in expected value for (33) if for each solution of (33a) there are no finite escape times almost surely and there exist $\alpha_{1}, \alpha_{2} \in \mathcal{K}_{\infty}$, and $\lambda>0$, such that, for each $x \in \mathbb{R}^{n}, \mathbf{x} \in \mathcal{S}_{\mathbf{r}}(x)$, and $(\mathbf{T}, \mathbf{J}) \in \mathrm{hst}(\mathbf{x})$, we have

$$
\mathbb{E}\left[\exp (\lambda(\mathbf{T}+\mathbf{J})) \alpha_{1}\left(|\mathbf{x}(\mathbf{T}, \mathbf{J})|_{\mathcal{A}}\right)\right] \leqslant \alpha_{2}\left(|x|_{\mathcal{A}}\right) .
$$

This definition does not correspond to true exponential stability; indeed, in the non-stochastic case it is know that simple asymptotic stability is equivalent to the existence of $\alpha_{1}, \alpha_{2} \in$ $\mathcal{K}_{\infty}$ and $\lambda>0$ such that $\exp (\lambda(t+j)) \alpha_{1}\left(|x(t, j)|_{\mathcal{A}}\right) \leqslant$ $\alpha_{2}\left(|x(0,0)|_{\mathcal{A}}\right)$ for all solutions $x$ and all $(t, j) \in \operatorname{dom}(x)$. See [37, Prop. 7] for example. However, it is reasonable to drop the modifier "quasi-" when $\alpha_{1}(s), \alpha_{2}(s) \propto s^{p}$ for some $p \geqslant 1$.

We also note that if

$$
\mathbb{E}\left[\exp (\lambda(\mathbf{T}+\mathbf{J}))|\mathbf{x}(\mathbf{T}, \mathbf{J})|_{\mathcal{A}}^{q p}\right] \leqslant k|x|_{\mathcal{A}}^{q p}
$$

with $p \geqslant 1$ and $q>1$ then, by convexity of the function $s \mapsto s^{q}$ and Jensen's inequality, it follows that

$$
\mathbb{E}\left[\exp ((\lambda / q)(\mathbf{T}+\mathbf{J}))|\mathbf{x}(\mathbf{T}, \mathbf{J})|_{\mathcal{A}}^{p}\right] \leqslant k^{1 / q}|x|_{\mathcal{A}}^{p}
$$

In some situations, the set $\mathcal{A}$ is not stable in expected value but the expected value of an exponentially weighted function of the distance to $\mathcal{A}$ minus a positive offset is bounded. This situation is captured in the following definition. The closed set $\mathcal{A} \subset \mathbb{R}^{n}$ is said to be uniformly globally practically quasi-exponentially stable in expected value for (33) if for each solution of (33a) there are no finite escape times almost surely and there exist $\alpha_{1}, \alpha_{2} \in \mathcal{K}_{\infty}, \lambda>0$, and $c \geqslant 0$ such that, for each $\tau \geqslant 0, x \in \mathbb{R}^{n}, \mathbf{x} \in \mathcal{S}_{\mathbf{r}}(x)$, and with $(\mathbf{T}, \mathbf{J}):=\left(\mathbf{T}_{\dashv} \wedge \mathbf{T}_{\tau}, \mathbf{J}_{\dashv} \wedge \mathbf{J}_{\tau}\right)$ where $\left(\mathbf{T}_{\dashv}, \mathbf{J}_{\dashv}\right)$ is defined in (42) and $\left(\mathbf{T}_{\tau}, \mathbf{J}_{\tau}\right)$ is defined in (45b), we have

$$
\begin{array}{r}
\mathbb{E}\left[\exp (\lambda(\mathbf{T}+\mathbf{J}))\left(\alpha_{1}\left(|\mathbf{x}(\mathbf{T}, \mathbf{J})|_{\mathcal{A}}\right)-c\right)\right] \\
\leqslant \alpha_{2}\left(|x|_{\mathcal{A}}\right)-c .
\end{array}
$$

The bound (50) has implications for the probability that the distance of the solution to the set $\mathcal{A}$ is large after a prescribed amount of time. Recalling the definition of $\left(\mathbf{T}_{\tau}, \mathbf{J}_{\tau}\right)$ in (45b), the bound (50) implies that

$$
\begin{aligned}
& \mathbb{P}\left[\left(\mathbf{T}_{\tau}, \mathbf{J}_{\tau}\right) \in \operatorname{dom}(\mathbf{x}), \quad\left|\mathbf{x}\left(\mathbf{T}_{\tau}, \mathbf{J}_{\tau}\right)\right|_{\mathcal{A}} \geqslant M\right] \\
& \quad \leqslant \exp (-\lambda \tau) \frac{\alpha_{2}\left(|x|_{\mathcal{A}}\right)}{\alpha_{1}(M)}+c\left(\frac{\exp (\lambda)-\exp (-\lambda \tau)}{\alpha_{1}(M)}\right) .
\end{aligned}
$$

In particular, this probability tend to zero as $M$ tends to infinity or, in the case where $c=0$, when $\tau$ tends to infinity.

\section{SUFFICIENT CONDITIONS FOR STABILITY}

\section{A. Introduction}

For technical reasons, in this section we focus on Lyapunov-based stability conditions for bounded sets. Conditions for unbounded sets are considered in [2] for the special case $B(x) \equiv 0$. Also, for simplicity and borrowing from [2] and [5] with inspiration from [15], we impose the following basic conditions on the data of (33):

Assumption 1: (Stochastic hybrid basic conditions)

1) $C, D \subset \mathbb{R}^{n}$ are closed;

2) $F: \mathbb{R}^{n} \rightrightarrows \mathbb{R}^{n}$ is outer semicontinuous ${ }^{11}$ and locally bounded $^{12}$ with nonempty convex values on $C$;

3) a) $G: \mathbb{R}^{n} \times \mathbb{R}^{m} \rightrightarrows \mathbb{R}^{n}$ is locally bounded,

b) $v \mapsto \operatorname{graph}(G(\cdot, v)):=\left\{(x, y) \in \mathbb{R}^{2 n}: y \in G(x, v)\right\}$ is measurable ${ }^{13}$ (with respect to the Borel $\sigma$ algebra on $\mathbb{R}^{m}$ ) with closed values.

4) $B: \mathbb{R}^{n} \rightrightarrows \mathbb{R}^{n \times r}$ is outer semicontinuous, locally bounded and, for each $x \in C, B(x)$ is nonempty, convex and diagonally convex, i.e., the set $\left\{\sigma \in \mathbb{R}^{n \times n}: \sigma=b b^{\top}, b \in B(x)\right\}$ is convex.

In all that follows $\mathcal{V}:=\cup_{\omega \in \Omega, i \in \mathbb{Z}_{\geqslant 1}} \mathbf{v}_{i}(\omega)$.

A function $V: \operatorname{dom}(V) \rightarrow \mathbb{R}$ is a certification candidate for $\mathcal{H}=(C,(F, B), D, G, \mu)$, and we write $V \in \mathcal{D}(\mathcal{H})$, if

C1. $C \cup D \cup G(D \times \mathcal{V}) \subset \operatorname{dom}(V)$,

C2. $0 \leqslant V(x)$ for all $x \in C \cup D \cup G(D \times \mathcal{V})$,

C3. the quantity $\int_{\mathbb{R}^{m}} \sup _{g \in G(x, v)} V(g) \mu(d v)$ is well defined (and finite) for each $x \in D$, using the convention that $\sup _{g \in G(x, v)} V(g)=0$ when $G(x, v)=\varnothing$, justified by the preceding item $\mathrm{C} 2$, and

C4. $V$ is twice continuously differentiable on an open set containing $C$.

Under the third item of Assumption 1, if $V: \operatorname{dom}(V) \rightarrow$ $\mathbb{R}$ is upper semicontinuous then C1-C2 imply C3. See [2, Lemma 4.1].

Lyapunov-based sufficient conditions for asymptotic stability and recurrence are expressed in terms of the following operators on certification candidates. Given a closed set $S \subset \mathbb{R}^{n}$, let $V: \operatorname{dom} V \rightarrow \mathbb{R}$ be a certification candidate for $\mathcal{H}_{\cap S}:=(C \cap S,(F, B), D \cap S, G \cap S, \mu)$. For all $x \in C \cap S$

\footnotetext{
${ }^{11}$ See Footnote 5.

${ }^{12}$ See Footnote 6.

${ }^{13}$ See Footnote 9.
} 
define

$$
\begin{aligned}
& \mathcal{L}_{S} V(x):= \\
& \sup _{f \in F(x), b \in B(x)}\left(\langle\nabla V(x), f\rangle+\frac{1}{2} \operatorname{trace}\left(b b^{\top} \nabla^{2} V(x)\right)\right)
\end{aligned}
$$

and for all $x \in D \cap S$ define

$$
\Delta_{S} V(x):=\int_{\mathbb{R}^{m}} \sup _{g \in G(x, v) \cap S} V(g) \mu(d v)-V(x) .
$$

These quantities are well-defined and finite for each $x \in$ $C \cap S$, respectively $x \in D \cap S$, under the stochastic hybrid basic conditions of Assumption 1.

According to [5, Lemmas 8 \& 9], an analogue of Dynkin's formula applies to the solutions of (33). As a consequence, under Assumption 1, if $S \subset \mathbb{R}^{n}$ is closed, $V \in \mathcal{D}\left(\mathcal{H}_{\cap S}\right)$, $\mathbf{x} \in \mathcal{S}_{\mathbf{r}}\left(\mathbb{R}^{n}\right),(\mathbf{T}, \mathbf{J}) \in \operatorname{hst}(\mathbf{x}), \mathbb{E}[\mathbf{T}]<\infty, \mathbf{J}$ is uniformly bounded almost surely, and either $V$ has compact support or $(\mathbf{T}, \mathbf{J})$ is bounded by the first exit time from a compact set then

$$
\begin{aligned}
& \mathbb{E}[V(\mathbf{x}(\mathbf{T}, \mathbf{J}))]-V(x) \\
& \leqslant \mathbb{E}\left[\sum_{i=0}^{\mathbf{J}-1} \Delta_{S} V\left(\mathbf{x}\left(\mathbf{T}_{i+1}, i\right)\right)+\int_{0}^{\mathbf{T}} \mathcal{L}_{S} V\left(\mathbf{x}\left(s, \mathbf{J}_{s}\right)\right) d s\right]
\end{aligned}
$$

where $\mathbf{J}_{s}$ and $\mathbf{T}_{i+1}$ were defined at the end of Section VIIIB.

The inequality (53) plays a fundamental role in establishing almost all of the upcoming sufficient conditions for asymptotic stability, recurrence, and stability in expected value. In Section XII, the results that appear subsequently will be demonstrated in the context of the examples introduced in Section VII.

\section{B. Stability and uniform asymptotic stability}

The following theorem, providing sufficient conditions of uniform global stability in probability, is taken from [5, Theorem 3]; see also [2, Theorem 4.2] for the case where $B(x) \equiv 0$.

Theorem 5: For the stochastic hybrid system $\mathcal{H}$ in (33) under Assumption 1, the compact set $\mathcal{A} \subset \mathbb{R}^{n}$ is uniformly globally stable in probability if there exist $\alpha_{1}, \alpha_{2} \in \mathcal{K}_{\infty}$ and $V \in \mathcal{D}(\mathcal{H})$ such that

$$
\begin{array}{rlrl}
\alpha_{1}\left(|x|_{\mathcal{A}}\right) & \leqslant V(x) & & \forall x \in C \cup D \cup G(D \times \mathcal{V}) \\
V(x) & \leqslant \alpha_{2}\left(|x|_{\mathcal{A}}\right) & & \forall x \in C \cup D \\
\mathcal{L}_{\mathbb{R}^{n}} V(x) \leqslant 0 & & \forall x \in C \\
\Delta_{\mathbb{R}^{n}} V(x) & \leqslant 0 & & \forall x \in D
\end{array}
$$

where $\mathcal{L}_{\mathbb{R}^{n}} V(x)$ and $\Delta_{\mathbb{R}^{n}} V(x)$ are defined in (51) and (52), respectively.

A similar result, which is relevant for recurrence studies, is the following theorem, taken from [5, Thm. 4].

Theorem 6: For the stochastic hybrid system $\mathcal{H}$ in (33) under Assumption 1, the closure of the open, bounded set $\mathcal{O} \subset \mathbb{R}^{n}$ is uniformly Lagrange stability in probability conditioned on stopping at $\mathcal{O}$ if there exist $\alpha_{1}, \alpha_{2} \in \mathcal{G}_{\infty}$ and $V \in \mathcal{D}\left(\mathcal{H}_{\cap O^{c}}\right)$ (where $\mathcal{O}^{c}:=\mathbb{R}^{n} \backslash \mathcal{O}$ ) such that

$$
\begin{array}{rlrl}
\alpha_{1}\left(|x|_{\overline{\mathcal{O}}}\right) \leqslant V(x) & \forall x \in & (C \cup D \cup G(D \times \mathcal{V})) \cap \mathcal{O}^{c} \\
V(x) \leqslant \alpha_{2}\left(|x|_{\overline{\mathcal{O}}}\right) & & \forall x \in(C \cup D) \cap \mathcal{O}^{c} & \\
\mathcal{L}_{\mathcal{O}^{c}} V(x) \leqslant 0 & \forall x \in C \cap \mathcal{O}^{c} \\
\Delta_{\mathcal{O}^{c}} V(x) \leqslant 0 & \forall x \in D \cap \mathcal{O}^{c} .
\end{array}
$$

where $\mathcal{L}_{\mathcal{O}^{c}} V(x)$ and $\Delta_{\mathcal{O}^{c}} V(x)$ are defined in (51) and (52), respectively.

In the next statement, given a compact set $\mathcal{A} \subset \mathbb{R}^{n}, \rho \in$ $\mathcal{P D}(\mathcal{A})$ if $\rho: \mathbb{R}^{n} \rightarrow \mathbb{R}_{\geqslant 0}$ is continuous and $\rho(x)=0$ if and only if $x \in \mathcal{A}$. The main result for uniform global asymptotic stability is the following theorem, taken from [5, Corollary 1].

Theorem 7: For the stochastic hybrid system $\mathcal{H}$ in (33) under Assumption 1, the compact set $\mathcal{A} \subset \mathbb{R}^{n}$ is uniformly globally asymptotically stable in probability if there exist $V \in \mathcal{D}(\mathcal{H})$ and $\rho \in \mathcal{P} \mathcal{D}(\mathcal{A})$ such that (54a)-(54b) hold and

$$
\begin{aligned}
\mathcal{L}_{\mathbb{R}^{n}} V(x) \leqslant-\rho(x) & & \forall x \in C \\
\Delta_{\mathbb{R}^{n}} V(x) \leqslant-\rho(x) & & \forall x \in D .
\end{aligned}
$$

The conditions of the previous theorem can be relaxed somewhat in the case where $V$ is continuous on its domain. The following result is established following the technique used to establish [2, Thm. 5.1].

Theorem 8: For the stochastic hybrid system $\mathcal{H}$ in (33) under Assumption 1, the compact set $\mathcal{A} \subset \mathbb{R}^{n}$ is uniformly globally asymptotically stable in probability if there exist $V \in \mathcal{D}(\mathcal{H})$ such that the conditions of Theorem 5 hold and, in addition, $V$ is continuous on its domain and for each compact set $K \subset \mathbb{R}^{n}$ satisfying $K \cap \mathcal{A}=\varnothing$,

$$
\begin{array}{llrl}
\mathcal{L}_{K} V(x)<0 & & \forall x \in C \backslash \mathcal{A} \\
\Delta_{K} V(x)<0 & & \forall x \in D \backslash \mathcal{A}
\end{array}
$$

where $\mathcal{L}_{K} V(x)$ and $\Delta_{K} V(x)$ are defined in (51) and (52), respectively.

We note that a sufficient condition for the inequalities (56) to hold for each compact set $K \subset \mathbb{R}^{n}$ is that they hold with $K$ replaced by $\mathbb{R}^{n}$. However, this latter condition is not necessary. For example, consider a purely discrete-time system with $g(x, v)=(0.5+v) x$ where $\mu$ is a distribution with mean 0 and variance 0.75 , as in $[34, \S 8.5 .3]$. Suppose further that $\mu(F)<1$ for each compact set $F \subset \mathbb{R}$. Define the certification candidate $V: \mathbb{R} \rightarrow \mathbb{R}$ via $V(x):=x^{2}$ for all $x \in \mathbb{R}$ and $\mathcal{A}:=\{0\}$. We get

$$
\begin{aligned}
\int_{\mathbb{R}} V(g(x, v)) \mu(d v) & =\int_{\mathbb{R}}((0.5+v) x)^{2} \\
& =x^{2}\left(0.25+\int_{\mathbb{R}} v^{2} \mu(d v)\right) \\
& =x^{2}=V(x) .
\end{aligned}
$$

Thus, (56b) does not hold with $K$ replaced by $\mathbb{R}$. 
On the other hand, for each compact set $K \subset \mathbb{R}$ that is disjoint from the origin and each $x \in \mathbb{R} \backslash\{0\}$, there exists a compact set $F \subset \mathbb{R}$ and $\beta \geqslant 1$ such that, with the definition $\mathcal{I}:=[-\beta, \beta]$,

$$
\begin{aligned}
& \int_{\mathbb{R}} \max _{\hat{g} \in\{g(x, v)\} \cap K} V(\hat{g}) \mu(d v)=\int_{F} V(g(x, v)) \mu(d v) \\
& \leqslant \int_{\mathcal{I}} V(g(x, v)) \mu(d v) \\
& <\int_{\mathcal{I}} V(g(x, v)) \mu(d v)+((0.5-\beta) x)^{2}(1-\mu(\mathcal{I})) \\
& \left.\leqslant \int_{\mathcal{I}} V(g(x, v)) \mu(d v)+\int_{\mathcal{I}^{c}} V(g, v)\right) \mu(d v) \\
& =\int_{\mathbb{R}} V(g(x, v)) \mu(d v)=V(x) .
\end{aligned}
$$

The strict inequality above, which establishes (56b), follows from the assumptions that $\mu(\mathcal{I}) \in[0,1)$ and $\beta \geqslant 1$, and the condition $x \neq 0$.

\section{Recurrence}

We now turn our attention to sufficient conditions for uniform global recurrence in probability. The next result is [5, Thm. 5].

Theorem 9: For the stochastic hybrid system $\mathcal{H}$ under Assumption 1 , the open set $\mathcal{O} \subset \mathbb{R}^{n}$ such that $\mathcal{O}^{c}$ is compact is uniformly globally recurrent in probability if there exist $\alpha \in \mathcal{G}_{\infty}, \varepsilon>0$, and $V \in \mathcal{D}\left(\mathcal{H}_{\cap \mathcal{O}^{c}}\right)$ such that

$$
\begin{aligned}
V(x) & \leqslant \alpha\left(|x|_{\overline{\mathcal{O}}}\right) & & \forall x \in(C \cup D) \cap \mathcal{O}^{c}, \\
\mathcal{L}_{\mathcal{O}^{c}} V(x) & \leqslant-\varepsilon & & \forall x \in C \cap \mathcal{O}^{c}, \\
\Delta_{\mathcal{O}^{c}} V(x) & \leqslant-\varepsilon & & \forall x \in D \cap \mathcal{O}^{c},
\end{aligned}
$$

where $\mathcal{L}_{\mathcal{O}^{c}} V(x)$ and $\Delta_{\mathcal{O}^{c}} V(x)$ are defined in (51) and (52), respectively.

In the next statement, which is [5, Corollary 2], given an open set $\mathcal{O} \subset \mathbb{R}^{n}, \rho \in \mathcal{P} \mathcal{D}(\mathcal{O})$ if $\rho: \mathbb{R}^{n} \rightarrow \mathbb{R}_{\geqslant 0}$ is continuous and satisfies $\rho(x)>0$ for all $x \in \mathbb{R}^{n} \backslash \mathcal{O}$.

Corollary 1: For the stochastic hybrid system $\mathcal{H}$ under Assumption 1 , the open set $\mathcal{O} \subset \mathbb{R}^{n}$ is uniformly globally recurrent in probability if there exist $V \in \mathcal{D}\left(\mathcal{H}_{\cap \mathcal{O}^{c}}\right)$ and $\rho \in \mathcal{P} \mathcal{D}(\mathcal{O})$ such that (55a)-(55b) hold and

$$
\begin{array}{ll}
\mathcal{L}_{\mathcal{O}^{c}} V(x) \leqslant-\rho(x) & \forall x \in C \cap \mathcal{O}^{c}, \\
\Delta_{\mathcal{O}^{c}} V(x) \leqslant-\rho(x) & \forall x \in D \cap \mathcal{O}^{c},
\end{array}
$$

where $\mathcal{L}_{\mathcal{O}^{c}} V(x)$ and $\Delta_{\mathcal{O}^{c}} V(x)$ are defined in (51) and (52), respectively.

\section{Asymptotic stability in expected value}

In this section, we provide Lyapunov-function-based sufficient conditions for uniform global stability and quasiexponential stability in expected value. These results have not appeared before in the stochastic hybrid inclusions literature but can be derived easily from the hybrid Dynkin's formula given in (53).
Theorem 10: The compact set $\mathcal{A} \subset \mathbb{R}^{n}$ is uniformly globally stable in expected value if the conditions of Theorem 5 hold.

Theorem 11: The compact set $\mathcal{A} \subset \mathbb{R}^{n}$ is uniformly globally quasi-exponentially stable in expected value if the conditions of Theorem 7 hold with $\rho \in \mathcal{P} \mathcal{D}(\mathcal{A})$ being such that there exists $\lambda>0$ satisfying $\lambda V(x) \leqslant \rho(x)$ for all $x \in \mathbb{R}^{n}$.

An analogous result holds for uniform global practical quasi-exponential stability.

Theorem 12: For the stochastic hybrid system $\mathcal{H}$ in (33) under Assumption 1, the compact set $\mathcal{A} \subset \mathbb{R}^{n}$ is uniformly globally practically quasi-exponentially stable in probability if there exist $V \in \mathcal{D}(\mathcal{H}), \lambda>0$ and $c \geqslant 0$ such that (54a)(54b) hold and

$$
\begin{array}{ll}
\mathcal{L}_{\mathbb{R}^{n}} V(x) \leqslant-\lambda V(x)+c & \forall x \in C \\
\Delta_{\mathbb{R}^{n}} V(x) \leqslant-\lambda V(x)+c & \forall x \in D .
\end{array}
$$

\section{E. Matrosov-Foster functions}

Sometimes it is difficult to find a single certification candidate satisfying the conditions for recurrence, or asymptotic stability, given in the previous sections. In this case, it is useful to know that it is sufficient to search for a family of functions, none of which satisfies the conditions of the previous sections but which, taken together, still permits a strong conclusion about recurrence or asymptotic stability.

We start by considering the situation where $\mathcal{O} \subset \mathbb{R}^{n}$ is open and its complement is compact. Let $m \in \mathbb{Z}_{\geqslant 1}$. Following [38] and [2, 55.2$]$, we say that the $m+1$ functions $V_{i}, i \in\{0, \ldots, m\}$ are Matrosov-Foster functions for $\mathcal{O}$ if there exist continuous functions $u_{c, i}: C \cap \mathcal{O}^{c} \rightarrow \mathbb{R}$ and $u_{d, i}: D \cap \mathcal{O}^{c} \rightarrow \mathbb{R}, i \in\{0, \ldots, m\}$, such that

1) for all $i \in\{0, \ldots, m\}, V_{i}$ is a certification candidate for $\left(C \cap \mathcal{O}^{c},(F, B), D \cap \mathcal{O}^{c}, G \cap \mathcal{O}^{c}, \mu\right)$ that is bounded on $(C \cup D) \cap \mathcal{O}^{c}$, and

$$
\begin{array}{ll}
\mathcal{L}_{\mathcal{O}^{c}} V_{i}(x) \leqslant u_{c, i}(x) & \forall x \in C \cap \mathcal{O}^{c}, \\
\Delta_{\mathcal{O}^{c}} V_{i}(x) \leqslant u_{d, i}(x) & \forall x \in D \cap \mathcal{O}^{c} ;
\end{array}
$$

2) a) $u_{c, 0}(x) \leqslant 0$ for all $x \in C \cap \mathcal{O}^{c}$,

b) $u_{d, 0}(x) \leqslant 0$ for all $x \in D \cap \mathcal{O}^{c}$,

c) with the functions $u_{c, m+1}, u_{d, m+1}: \mathbb{R}^{n} \rightarrow\{1\}$, for each $j \in\{0,1, \ldots, m\}$ we have

i) if $x \in C \cap \mathcal{O}^{c}$ and $u_{c, i}(x)=0$ for all $i \in$ $\{0,1, \ldots, j\}$ then $u_{c, j+1}(x) \leqslant 0$,

ii) if $x \in D \cap \mathcal{O}^{c}$ and $u_{d, i}(x)=0$ for all $i \in$ $\{0,1, \ldots, j\}$ then $u_{d, j+1}(x) \leqslant 0$.

As has been pointed out in the literature before (see [2, p. 10] for example), condition 2 amounts to a nested negative semi-definite condition on the functions $u_{c, i}$ and $u_{d, i}$. The first two items of condition 2 require that the functions $u_{c, 0}$ and $u_{d, 0}$ are never positive; the last item enforces that $u_{c, j+1}$ (respectively $u_{d, j+1}$ ) can be positive only where at least one of the functions $u_{c, i}, i \in\{0, \ldots, j\}$, is negative. Considering $j=m$ and the definition of $u_{c, m+1}$ (respectively, $u_{d, m+1}$ ), there are no points in $C \cap \mathcal{O}^{c}$ (respectively, $D \cap \mathcal{O}^{c}$ ) where 
all of the functions $u_{c, i}$ (respectively, $u_{d, i}$ ), $i \in\{0, \ldots, m\}$, are zero.

Matrosov-Foster functions can be used to establish uniform global recurrence of an open set or uniform global asymptotic stability in probability of a compact set, as indicated in the next two results which are based on [2, Corollaries $5.3 \& 5.4]$.

Theorem 13: Let $\mathcal{O} \subset \mathbb{R}^{n}$ be open and bounded. For the system (33), suppose Assumption 1 holds and that $\mathcal{O}$ is uniformly Lagrange stable in probability conditioned on stopping at $\mathcal{O}$. If for each $\Delta>0$ there exist MatrosovFoster functions for $\mathcal{O} \cup\left(\mathbb{R}^{n} \backslash(\overline{\mathcal{O}}+\Delta \mathbb{B})\right)$ for (33) then $\mathcal{O}$ is uniformly globally recurrent for (33).

Theorem 14: Let $\mathcal{A} \subset \mathbb{R}^{n}$ be compact. Suppose Assumption 1 holds and that $\mathcal{A}$ is uniformly globally stable in probability for (33). If for each pair of positive real numbers $(\delta, \Delta)$, there exist Matrosov-Foster functions for $\left(\mathcal{A}+\delta \mathbb{B}^{\circ}\right) \cup\left(\mathbb{R}^{n} \backslash(\mathcal{A}+\Delta \mathbb{B})\right)$ for (33) then $\mathcal{A}$ is uniformly globally asymptotically stable in probability for (33).

These results will be demonstrated by one of the examples reconsidered in Section XII.

\section{RESUlts BASED ON SEQUENTIAL COMPACTNESS FOR SYSTEMS WITH NON-STOCHASTIC FLOWS}

\section{A. Introduction}

Regarding stability theory for non-stochastic hybrid systems, there are several powerful aspects that follow from the fact that the set of solutions has a sequential compactness property under the regularity conditions listed at the end Section VI-B. Such systems are examples of what are sometimes called "well-posed" hybrid systems [15, Ch. 6]. One consequence is that asymptotic stability of a compact set for a well-posed hybrid system is robust to sufficiently small inner and outer state perturbations [18, Thm. 6.6], [15, §7.3]. This fact is related to the observation, again a consequence of sequential compactness, that the reachable set in finite time under small perturbations is contained in a small perturbation of the reachable set without perturbations [18, Corollary 5.5], [15, Prop. 6.34]. An ensuing corollary is that well-posed hybrid systems with an asymptotically stable compact set or globally recurrent open bounded set admit smooth Lyapunov functions [30], [33]. Another consequence is that asymptotic stability is equivalent to uniform asymptotic stability for a compact attractor [18, Prop. 6.2, Thm. 6.5], [15, Thm. 7.12], and that global recurrence is equivalent to uniform global recurrence for an open bounded set [33]. In addition, uniform attractivity of a forward invariant compact set implies that the set is stable and thus uniformly asymptotically stable [18, Prop. 6.1], [15, Prop. 7.5]. Finally, we mention that sequential compactness enables the classical invariance principle of Krasovskii and LaSalle to be established for well-posed non-stochastic hybrid systems [39], [15, Ch. 8], which is often used to relax Lyapunov-based sufficient conditions for asymptotic stability or recurrence.

For stochastic hybrid systems of the form (33), a type of sequential compactness result has been established in [3, Thm. 1] under Assumption 1 for the special case where
$B(x) \equiv 0$. Such a result for the case where Brownian motion is present remains an open problem. The sequential compactness result in [3] enables several observations that parallel the results discussed above for non-stochastic hybrid systems. For example, statements can be made about reachability and robust reachability [3, Thms. $3 \& 4$, uniform attractivity implying stability [3, Thm. 5], almost sure recurrence implying uniform recurrence in probability [3, Thm. 6] and thus almost sure attractivity implying uniform attractivity in probability [3, Corollary 2], that recurrence is robust to sufficiently small state perturbations [40], and that an invariance principle applies and can be used to relax Lyapunov-based sufficient conditions for asymptotic stability [4], [6]. Such results are summarized in the following subsections. Results pertaining to robustness of asymptotic stability in probability, as well as converse Lyapunov theorems, are currently under development.

\section{B. Reachability and perturbed reachability}

Here we recall a result on the nature of finite-time reachable sets from compact sets for the case where $B(x) \equiv 0$. The result, [3, Thm. 4], is like the result for non-stochastic hybrid systems in [18, Theorem 4.6] which establishes that, in the absence of finite escape times, the finite-time reachable set from any compact set is compact.

Theorem 15: Consider the system (33) with $B(x) \equiv 0$ under Assumption 1. If there are no finite escape times for (33a) then, for each compact $K_{1} \subset \mathbb{R}^{n}, \varrho>0$, and $\tau>0$ there exists a compact set $K_{2}$ such that, for each $\mathbf{x} \in \mathcal{S}_{\mathbf{r}}\left(K_{1}\right)$,

$$
\mathbb{P}\left(\operatorname{graph}_{\leqslant \tau}(\mathbf{x}) \subset \mathbb{R}^{2} \times K_{2}\right) \geqslant 1-\varrho .
$$

Next we recall a result on a type of robustness of the finitetime reachable set under perturbations of initial conditions. The result appears as [3, Thm. 3].

Theorem 16: Consider the system (33) with $B(x) \equiv 0$ under Assumption 1. Let $K_{0} \subset K \subset \mathbb{R}^{n}$ be compact, $\tau>0$ and $\varrho \geqslant 0$. If, for each $\mathbf{x} \in \mathcal{S}_{\mathbf{r}}\left(K_{0}\right)$,

$$
\mathbb{P}\left(\operatorname{graph}_{\leqslant \tau}(\mathbf{x}) \subset \mathbb{R}^{2} \times K\right) \geqslant 1-\varrho
$$

then, for each $\varepsilon>0$, there exists $\delta>0$ such that, for each $\mathbf{x} \in \mathcal{S}_{\mathbf{r}}\left(K_{0}+\delta \mathbb{B}^{\circ}\right)$,

$$
\mathbb{P}\left(\operatorname{graph}_{\leqslant \tau}(\mathbf{x}) \subset\left(\mathbb{R}^{2} \times\left(K+\varepsilon \mathbb{B}^{\circ}\right)\right)\right) \geqslant 1-\varrho-\varepsilon .
$$

C. Almost sure forward invariance plus UGAp implies $U G A S p$

A compact set $K \subset \mathbb{R}^{n}$ is said to be almost surely strongly forward invariant if, for each $\mathbf{x} \in \mathcal{S}_{\mathbf{r}}(K), \operatorname{graph}(\mathbf{x}(\omega)) \subset$ $\mathbb{R}^{2} \times K$ for almost all $\omega \in \Omega$, i.e., $\mathbb{P}\left(\operatorname{graph}(\mathbf{x}) \subset \mathbb{R}^{2} \times K\right)=$ 1 .

The next result is a stochastic version of [18, Propositions $6.1 \& 6.3]$, specialized to the case where the basin of attraction is the entire state space. It uses Theorem 16, which relied on the sequential compactness result described in the introduction of this section.

Theorem 17: Consider the system (33) with $B(x) \equiv 0$ under Assumption 1. The compact set $\mathcal{A}$ is uniformly globally asymptotically stable in probability if and only if it 
is almost surely strongly forward invariant and uniformly globally attractive in probability.

\section{Uniformity of recurrence}

An open set $\mathcal{O}$ is said to be almost surely globally recurrent if there are no finite escape times for $x \in C, \dot{x} \in$ $F(x)$ and, for each $\mathbf{x} \in \mathcal{S}_{\mathbf{r}}\left(\mathbb{R}^{n}\right)$ and for almost every $\omega \in \Omega$ if $\mathbf{x}(\omega)$ is complete then $\mathbf{x}(\omega)$ reaches $\mathcal{O}$, that is if $\mathbf{x}(\omega)$ is complete then $\operatorname{graph}(\mathbf{x}(\omega)) \cap\left(\mathbb{R}^{2} \times \mathcal{O}\right) \neq \varnothing$.

The following result appears as [2, Prop. 3.1] and [3, Thm. $6]$.

Theorem 18: An open bounded set is almost surely globally recurrent if and only if it is uniformly globally recurrent in probability.

As noted in explaining [3, Corollary 2], the combination of Theorem 18, Theorem 17, and [2, Proposition 2.2] yields the following corollary, which is the stochastic version of [18, Proposition 6.2].

Corollary 2: If the compact set $\mathcal{A}$ is Lyapunov stable in probability and each open neighborhood of $\mathcal{A}$ is almost surely globally recurrent then $\mathcal{A}$ is uniformly globally asymptotically stable in probability.

\section{E. Robustness}

Robustness pertains to maintaining a stability property even when the data of the stochastic hybrid system in (33) is inflated to $\mathcal{H}_{\delta}:=\left(C_{\delta}, F_{\delta}, D_{\delta}, G_{\delta}, \mu\right)$ where $\delta: \mathbb{R}^{n} \rightarrow \mathbb{R}_{\geqslant 0}$ continuous and

$$
\begin{aligned}
C_{\delta} & :=\left\{x \in \mathbb{R}^{n}:(x+\delta(x) \mathbb{B}) \cap C \neq \varnothing\right\} \\
F_{\delta} & :=\overline{\operatorname{co}} F((x+\delta(x) \mathbb{B}) \cap C)+\delta(x) \mathbb{B} \\
D_{\delta} & :=\left\{x \in \mathbb{R}^{n}:(x+\delta(x) \mathbb{B}) \cap D \neq \varnothing\right\} \\
G_{\delta} & :=\left\{g \in \mathbb{R}^{n}: g=\tilde{g}+\delta(g) \mathbb{B}, \tilde{g} \in G((x+\delta(x) \mathbb{B}) \cap D, v)\right\},
\end{aligned}
$$

The following statement, about robustness of global recurrence, is established in [40].

Theorem 19: Under Assumption 1, if an open bounded set $\mathcal{O} \subset \mathbb{R}^{n}$ is globally recurrent for (33) then there exists a continuous function $\delta: \mathbb{R}^{n} \rightarrow \mathbb{R}_{>0}$ such that $\mathcal{O}$ is uniformly globally recurrent for $\mathcal{H}_{\delta}$.

Robustness results for uniform global asymptotic stability in probability are currently under development.

\section{F. Invariance/recurrence principle-based stability results}

Based on the results in [26], the papers [4], [6] establishes a recurrence principle, which generalizes the classical invariance principle, for the system (33) with $B(x) \equiv 0$. When applied to the study of asymptotic stability or recurrence, the principle yields the following results, which are contained in [6, Thms. $2 \& 3]$.

Theorem 20: Suppose the conditions of Theorem 5 hold for the system (33) with $B(x) \equiv 0$ under Assumption 1. Then $\mathcal{A}$ is UGASp if and only if there does not exist an almost surely complete solution that remains in a non-zero level set of $V$ almost surely.

Theorem 21: Suppose the conditions of Theorem 6 hold hold for the system (33) with $B(x) \equiv 0$ under Assumption 1.
Then $\mathcal{O}$ is uniformly globally recurrent in probability if and only if there does not exist an almost surely complete solution that remains almost surely in the set $L_{V}(c) \cap\left(\mathbb{R}^{n} \backslash \mathcal{O}\right)$ for some $c \geqslant 0$.

These results provide an alternative to the use of MatrosovFoster functions for establishing UGASp or UGRp, as in Section X-E. The advantage of Theorems 20 and 21 is that they do not requiring finding Matrosov-Foster functions; their disadvantage is that they require some knowledge about the behavior of solutions.

\section{THE EXAMPLES REVISITED: STABIITY ANALYSIS}

\section{A. Sampled-data stochastic control systems}

We consider stability properties relative to the compact set $\mathcal{A}:=\{0\} \times[0, T]$.

Suppose there exists a smooth matrix-valued function $P$ : $[0, T] \rightarrow \mathbb{R}^{n \times n}$ and strictly positive real numbers $c_{1}, c_{2}, \varepsilon$ and $c \geqslant 0$ such that, for all $\tau \in[0, T]$ and $z \in \mathbb{R}^{n}, P(\tau)=$ $P^{\top}(\tau)$,

$$
\begin{gathered}
c_{1} I \leqslant P(\tau) \leqslant c_{2} I \\
z^{\top} \dot{P}(\tau) z+z^{\top} P(\tau) f(z)+f^{\top}(z) P(\tau) z \\
+0.5 \operatorname{trace}\left(b(z) b(z)^{\top} P(\tau)\right) \leqslant-\varepsilon z^{\top} z+c \\
g^{\top}(z) P(0) g(z)-g^{\top}(z) P(T) g(z) \leqslant-\varepsilon z^{\top} z+c .
\end{gathered}
$$

The ensuing claims then follow using the certification candidate $V(x):=z^{\top} P(\tau) z$. When $c=0$, it follows from Theorems 7 and 11 respectively that the set $\mathcal{A}$ is uniformly globally asymptotically stable in probability and uniformly globally exponentially ${ }^{14}$ stable in expected value. When $c>$ 0 , it follows from Corollary 1 and Theorem 12 respectively that each open set containing $\left\{z \in \mathbb{R}^{n}: z^{\top} z \leqslant c / \varepsilon\right\} \times[0, T]$ is uniformly globally recurrent in probability and that $\mathcal{A}$ is uniformly globally practically exponentially stable in expected value.

Regarding the conditions (60), in the non-stochastic case and with linear mapping $f(z):=A z$ and $g(z):=J z$, the conditions hold with $c=0$ if and only if all eigenvalues of the matrix $\exp (A T) J$ have magnitude less than one, in which case one can take

$$
P(\tau):=\exp (-\widetilde{\varepsilon} \tau) \exp \left(A^{\top}(T-\tau)\right) X \exp (A(T-\tau)
$$

where $X=X^{\top}>0$ and $\tilde{\varepsilon}>0$ satisfy

$$
(\exp (A T) J)^{\top} X \exp (A T) J-\exp (-\widetilde{\varepsilon} T) X \leqslant-\widetilde{\varepsilon} I .
$$

B. Average dwell-time switching among stochastic differential equations

As an indication of the results that can be obtained for systems with average dwell-time switching among stochastic differential equations, consider the situation where, for each $q \in \mathcal{Q}$ there exist a twice continuously differentiable function $W_{q}: \mathbb{R}^{m} \rightarrow \mathbb{R}_{\geqslant 0}, \alpha_{1 q}, \alpha_{2 q} \in \mathcal{K}_{\infty}, \lambda_{q}>0, c_{q} \geqslant 0$ and $m_{q}>0$ such that, for all $(\xi, q) \in \mathbb{R}^{m} \times \mathcal{Q}$,

$$
\alpha_{1 q}(|\xi|) \leqslant W_{q}(\xi) \leqslant \alpha_{2 q}(|\xi|)
$$

\footnotetext{
${ }^{14}$ We drop the modifier "quasi" here since we can take $\alpha_{1}(s)=$ $\alpha_{2}(s) \propto s^{2}$.
} 
and

$$
\begin{array}{r}
\left\langle\nabla W_{q}(\xi), f_{q}(\xi)\right\rangle+\frac{1}{2} \operatorname{trace}\left(b_{q}(\xi) b_{q}(\xi)^{\top} \nabla^{2} W_{q}(\xi)\right) \\
\leqslant-\lambda_{q} W_{q}(\xi)+c_{q}
\end{array}
$$

and

$$
W_{q}(\xi) \leqslant m_{q} W_{p}(\xi) \quad \forall p \in \mathcal{Q}_{q} \backslash\{q\} .
$$

We assume

$$
m_{q}<\exp \left(\frac{\lambda_{q}}{\delta}\right) .
$$

Define $\mathcal{A}:=\{0\} \times \mathcal{Q} \times[0, N] \subset \mathbb{R}^{m} \times \mathbb{R} \times \mathbb{R}$. and consider the certification candidate $V: \mathbb{R}^{m+2} \rightarrow \mathbb{R}_{\geqslant 0}$ defined by

$$
V(x):=\exp \left(\rho_{q} \tau\right) W_{q}(\xi)
$$

where $\rho_{q}>0$ for each $q \in \mathcal{Q}$. Define $\rho:=\max _{q \in \mathcal{Q}} \rho_{q}$ and, for all $s \geqslant 0, \alpha_{1}(s):=\min _{q \in \mathcal{Q}} \alpha_{1 q}(s)$ and $\alpha_{2}(s):=$ $\max _{q \in \mathcal{Q}} \alpha_{2 q}(s)$. It follows from (61) that, for all $x \in C \cup$ $D \cup G(D)$,

$$
\alpha_{1}\left(|x|_{\mathcal{A}}\right) \leqslant V(x) \leqslant \exp (\rho N) \alpha_{2}\left(|x|_{\mathcal{A}}\right) .
$$

It is not difficult to verify that

$$
\begin{aligned}
& \mathcal{L}_{\mathbb{R}^{m+2}} V(x)=\rho_{q} \delta \exp \left(\rho_{q} \tau\right) W_{q}(\xi)+ \\
& \exp \left(\rho_{q} \tau\right)\left(\left\langle\nabla W_{q}(\xi), f_{q}(\xi)\right\rangle+\frac{1}{2} \operatorname{tr}\left(b_{q}(\xi) b_{q}(\xi)^{\top} \nabla^{2} W_{q}(\xi)\right)\right) \\
& \leqslant \exp \left(\rho_{q} \tau\right)\left(\left(\rho_{q} \delta-\lambda_{q}\right) W_{q}(\xi)+c_{q}\right) \\
& \leqslant\left(\rho_{q} \delta-\lambda_{q}\right) V(x)+\exp \left(\rho_{q} N\right) c_{q} .
\end{aligned}
$$

Moreover,

$$
\Delta_{\mathbb{R}^{m+2}} V(x)=\left(\exp \left(-\rho_{q}\right) m_{q}-1\right) V(x) .
$$

The condition (64) enables picking $\rho_{q} \in\left(0, \lambda_{q} / \delta\right)$ so that, for some $\varepsilon>0$ and $\tilde{c} \geqslant 0$, we have

$$
\begin{aligned}
& \mathcal{L}_{\mathbb{R}^{n}} V(x) \leqslant-\varepsilon V(x)+\widetilde{c} \\
& \Delta_{\mathbb{R}^{n}} V(x) \leqslant-\varepsilon V(x) .
\end{aligned}
$$

It follows that when $\tilde{c}=0$, which is the case when $c_{q}=0$ for all $q \in \mathcal{Q}$, that all of the conditions provided in Theorems 7, 10, and 11, respectively, for asymptotic stability in probability, and stability and quasi-exponential stability in expected value of the compact set $\mathcal{A}$ are satisfied. When $\widetilde{c}>0$, the conditions provided for recurrence in Corollary 1 are satisfied for any open set containing the set $\{x \in C \cup D: V(x) \leqslant \widetilde{c} / \varepsilon\}$. Morever, the conditions for practical quasi-exponential stability in expected value in Theorem 12 are satisfied.

\section{A stochastic bouncing ball system}

Let $\mathcal{A}$ be the origin in $\mathbb{R}^{2}$ and consider the certification candidate

$$
V(x)=\gamma x_{1}+\frac{1}{2} x_{2}^{2}
$$

It is not difficult to establish (see [15, Ex. 3.19]) that there exist $\alpha_{1}, \alpha_{2} \in \mathcal{K}_{\infty}$ such that

$$
\alpha_{1}(|x|) \leqslant V(x) \leqslant \alpha_{2}(|x|) \quad \forall x \in C \cup D \cup G(D) .
$$

We suppose that $\mathbf{v}_{i}: \Omega \rightarrow\{a, b\}$, where $0<a<1<b$, $\mu(\{a\})=p \in(0,1)$, and

$$
a^{2} p+b^{2}(1-p)<1 .
$$

It can be verified that

$$
\begin{array}{ll}
\mathcal{L}_{\mathbb{R}^{2}} V(x)=0 & \forall x \in C \\
\Delta_{\mathbb{R}^{2}} V(x)=-\left(1-a^{2} p-b^{2}(1-p)\right) V(x) & \forall x \in D .
\end{array}
$$$$
\forall x \in C \quad(71 \mathrm{a})
$$

It follows from (70) and Theorems 5 and 10, respectively, that the origin is uniformly globally stable in probability and uniformly globally stable in expected value (relative to the function $\alpha_{1}$ ), respectively.

In order to establish uniform global asymptotic stability in probability, there are multiple options.

One option involves invoking Theorem 20, which relies on the invariance principle. Indeed, every sample path of a solution that starts in a non-zero level set of $V$ has at least one jump and such a jump changes the value of $V$. There there does not exist an almost surely complete solution that remains in a non-zero level set of $V$ almost surely.

Another option involves invoking Theorem 14, with the Matrosov-Foster candidate functions $V_{1}(x):=V(x)$ and $V_{2}(x):=x_{2}$. These functions are bounded on each compact set and admit the bounds (58) for $\mathcal{O}^{c}=\{|x| \in[\delta, \Delta]\}$ with $0<\delta<\Delta<\infty$ with

$$
\begin{aligned}
& u_{c, 1}(x)=0 \\
& u_{c, 2}(x)=-\gamma
\end{aligned}
$$

and

$$
\begin{aligned}
& u_{d, 1}(x)=-\left(1-a^{2} p-b^{2}(1-p)\right) V(x) \\
& u_{d, 2}(x)=(a p+b(1-p)-1) x_{2} .
\end{aligned}
$$

It is straightforward to verify that these functions satisfy the conditions required to make $\left(V_{1}, V_{2}\right)$ Matrosov-Foster functions for each set $\delta \mathbb{B}^{\circ} \cup\left(\mathbb{R}^{2} \backslash \Delta \mathbb{B}\right)$. Thus, the origin is uniformly globally asymptotically stable in probability according to Theorem 14.

Finally, we note that the condition (70) can be relaxed by resorting to non-smooth certification candidates, as in [2, $\S 6.1]$.

\section{The nefarious nurse}

Define $\mathcal{A}:=\mathbb{S}^{1} \times\{0\} \subset \mathbb{R}^{3}$. We suppose

$$
\sigma_{g}+\sigma_{b}<2 \text {. }
$$

Consider the certification candidate $V(x):=x_{3}$. It is straightforward to verify that, for all $x \in C \cup D \cup G(D \times \mathcal{V})$, $V(x)=|x|_{\mathcal{A}}$. In addition, we have

$$
\begin{aligned}
\mathcal{L}_{\mathbb{R}^{3}} V(x) & =-\varepsilon V(x)+c & & \forall x \in C \\
\Delta_{\mathbb{R}^{3}} V(x) & =\left(0.5 \sigma_{g}+0.5 \sigma_{b}-1\right) V(x) & & \forall x \in D .
\end{aligned}
$$

When $c=0$, it follows from the condition (72) and Theorems 7 and 11 respectively that the set $\mathcal{A}$ is uniformly globally asymptotically stable in probability and uniformly globally 
exponentially $^{15}$ stable in expected value. When $c>0$, it follows from the condition (72) and Corollary 1 and Theorem 12 respectively that each open set containing $\mathbb{S}^{1} \times[0, c / \varepsilon]$ is uniformly globally recurrent in probability and that $\mathcal{A}$ is uniformly globally practically exponentially stable in expected value.

\section{OPEN PROBLEMS}

The preceding discussion about stochastic hybrid inclusions, as modeled in (33), suggests several open problems. The first gaping hole pertains to extending the results of Section XI to the case where the dispersion map $B$ is not identically zero. The main themes there include establishing 1) equivalence between uniform and non-uniform versions of asymptotic stability and recurrence, 2) inherent robustness of asymptotic stability and recurrence, 3) converse Lyapunov theorems for asymptotic stability and recurrence, and 4) an invariance principle that can be used to establish asymptotic stability and recurrence. Another pressing open problem is to find an efficient way to model spontaneous jumps with the most natural causality constraints so that the continuous-time infinitesimal generator in (51) includes terms related to spontaneous transitions, as in (22). Related ideas for stochastic hybrid inclusions with an identically zero dispersion map have been discussed in [2, §7]. It is likely also worthwhile to explore weaker stability definitions involving expected values and relaxed Lyapunov conditions that guarantee such properties. Finally, exploring applications that rely on stochastic hybrid inclusions is an important aspect that remains largely untouched.

\section{CONCLUSIONS}

Stochastic hybrid systems can be used to model a very rich class of dynamical behavior. They address systems where the states sometimes change continuously and sometimes change instantaneously, where each type of change can be affected by a random process, and where the timing of jumps can also be driven by a random process or be determined by the location of the state in the state space. Significant research on classes of stochastic hybrid systems appeared in the literature over thirty years ago, and some significant new developments have occurred over the last decade. Relatively simple models can be used to address systems that possess unique solutions and whose jumps are triggered spontaneously. Examples and stability theory for such systems were addressed in the first half of this tutorial. More complicated models and tools are needed for systems that admit non-unique solutions, where jumps can be triggered by the value of the state, and where there is no preference for continuous time over discrete time. A modeling framework that addresses these situations was described in the second half of this tutorial. Examples were also provided to illustrate the utility of such a modeling framework. Developments in this direction are relatively new, and several important open problems remain.

\footnotetext{
${ }^{15}$ We drop the modifier "quasi" here since we can take $\alpha_{1}(s)=\alpha_{2}(s)=$
}

\section{REFERENCES}

[1] J. P. Hespanha, "Modeling and analysis of networked control systems using stochastic hybrid systems," Annual Reviews in Control, vol. 38, no. 2, pp. 155-170, Oct. 2014.

[2] A. R. Teel, "Lyapunov conditions certifying stability and recurrence for a class of stochastic hybrid systems," Annual Reviews in Control, vol. 37, pp. 1-24, 2013.

[3] — - "On sequential compactness of solutions for a class of stochastic hybrid systems," in American Control Conference, 2014, pp. 45124517.

[4] — " "On a recurrence principle for a class of stochastic hybrid systems," in American Control Conference, 2014, pp. 4518-4523.

[5] _ , "Stochastic hybrid inclusions with diffusive flows," in IEEE 53rd Conference on Decision and Control, Dec 2014, pp. 3071-3076.

[6] A. Subbaraman and A. R. Teel, "A Krasovskii-LaSalle function based recurrence principle for a class of stochastic hybrid systems," in IEEE 53rd Conference on Decision and Control, Dec 2014, pp. 2310-2315.

[7] A. R. Teel, A. Subbaraman, and A. Sferlazza, "Stability analysis for stochastic hybrid systems: a survey," Automatica, vol. 50, no. 10, pp. 2435-2456, 2014.

[8] M. H. A. Davis, "Piecewise-deterministic Markov processes: A general class of non-diffusion stochastic models," Journal of the Royal Statistical Society. Series B (Methodological), vol. 46, no. 3, pp. pp. 353-388, 1984.

[9] M. Davis, Markov Models and Optimization, ser. Monographs on Statistics and Applied Probability. Chapman \& Hall, London, UK, 1993.

[10] T. Fujiwara and H. Kunita, "Stochastic differential equations of jump type and Lévy processes in diffeomorphisms group," J. Math. Kyoto Univ., vol. 25, no. 1, p. 71106, 1985.

[11] M. Mariton, Jump Linear Systems in Automatic Control. Marcel Dekker, New York, NY, 1990.

[12] M. Ghosh, A. Arapostathis, and S. Marcus, "Optimal control of switching diffusions with application to flexible manufacturing systems," SIAM J. Control Optim., vol. 31, no. 5, pp. 1183-1204, 1993.

[13] G. G. Yin and C. Zhu, Hybrid Switching Diffusions. Springer, 2010.

[14] J. Hu, J. Lygeros, and S. Sastry, "Towards a theory of stochastic hybrid systems," in Hybrid Systems: Computation and Control, N. Lynch and B. H. Krogh, Eds. Springer, 2000, pp. 160-173.

[15] R. Goebel, R. G. Sanfelice, and A. R. Teel, Hybrid Dynamical Systems. Princeton University Press, 2012.

[16] R. T. Rockafellar and R. J.-B. Wets, Variational Analysis. Springer, 1998.

[17] D. Antunes, J. P. Hespanha, and C. Silvestre, "Volterra integral approach to impulsive renewal systems: Application to networked control," IEEE Trans. Autom. Control, vol. 57, pp. 607-619, Mar. 2012.

[18] R. Goebel and A. R. Teel, "Solutions to hybrid inclusions via set and graphical convergence with stability theory applications," Automatica, vol. 42, pp. 573-587, 2006.

[19] R. Goebel, R. G. Sanfelice, and A. R. Teel, "Hybrid dynamical systems," IEEE Control Systems Magazine, vol. 29, no. 2, pp. 2893, April 2009.

[20] A. Subbaraman and A. R. Teel, "A converse Lyapunov theorem for strong global recurrence," Automatica, vol. 49, no. 10, pp. 2963 2974, 2013.

[21] — "A Matrosov theorem for strong global recurrence," Automatica, vol. 49 , no. 11 , pp. $3390-3395,2013$.

[22] S. Grammatico, A. Subbaraman, and A. R. Teel, "Discrete-time stochastic control systems: A continuous Lyapunov function implies robustness to strictly causal perturbations," Automatica, vol. 49, no. 10, pp. $2939-2952,2013$.

[23] A. R. Teel, "A Matrosov theorem for adversarial Markov decision processes," IEEE Transactions on Automatic Control, vol. 58, no. 8, pp. 2142-2148, Aug 2013.

[24] A. R. Teel, J. P. Hespanha, and A. Subbaraman, "A converse Lyapunov theorem and robustness for asymptotic stability in probability," IEEE Transactions on Automatic Control, vol. 59, no. 9, pp. 2426-2441, Sept 2014.

[25] — "Equivalent characterizations of input-to-state stability for stochastic discrete-time systems," IEEE Transactions on Automatic Control, vol. 59, no. 2, pp. 516-522, Feb 2014.

[26] A. R. Teel, "A recurrence principle for stochastic difference inclusions," IEEE Transactions on Automatic Control, vol. 60, no. 2, pp. 420-435, Feb 2015. 
[27] J.-P. Aubin, G. Da Prato, and H. Frankowska, "Stochastic invariance for differential inclusions," Set-Valued Analysis, vol. 8, no. 1-2, pp. 181-201, 2000.

[28] M. Kisielewicz, Stochastic Differential Inclusions and Applications. Springer, 2013.

[29] S. Mitra and D. Liberzon, "Stability of hybrid automata with average dwell time: an invariant approach," in 43rd IEEE Conference on Decision and Control, The Bahamas, December 2004, pp. 1394-1399.

[30] C. Cai, A. R. Teel, and R. Goebel, "Smooth Lyapunov functions for hybrid systems. Part II: (Pre-)asymptotically stable compact sets," IEEE Trans. Automat. Contr., vol. 53, no. 3, pp. 734 - 748, April 2008.

[31] J. P. Hespanha and A. S. Morse, "Stability of switched systems with average dwell-time," in Proc. 38th IEEE Conference on Decision and Control, Sidney, Australia, 1999, pp. 2655-2660.

[32] J. Lygeros, K. Johansson, S. Simić, J. Zhang, and S. S. Sastry, "Dynamical properties of hybrid automata," IEEE Trans. Autom. Control, vol. 48, no. 1, pp. 2-17, 2003.

[33] A. Subbaraman and A. R. Teel, "On the equivalence between global recurrence and the existence of a smooth Lyapunov function for hybrid systems," 2015, submitted.

[34] H. Kushner, Introduction to stochastic control. Holt, Rinehart, and Winston, Inc., 1971.

[35] S. P. Meyn and R. L. Tweedie, Markov Chains and Stochastic Stability. Springer-Verlag, 1993

[36] R. Z. Khasminskii, Stochastic Stability of Differential Equations. Sijthoff \& Noordhoff, Netherlands, 1980.

[37] E. D. Sontag, "Comments on integral variants of ISS," Systems \& Control Letters, vol. 34, no. 1, pp. 93-100, 1998.

[38] R. G. Sanfelice and A. R. Teel, "Asymptotic stability in hybrid systems via nested Matrosov functions," IEEE Trans. Autom. Control, vol. 54 no. 7, pp. 1569-1574, 2009.

[39] R. G. Sanfelice, R. Goebel, and A. R. Teel, "Invariance principles for hybrid systems with connections to detectability and asymptotic stability," IEEE Trans. Autom. Control, vol. 52, no. 12, pp. 2282-2297, December 2007.

[40] A. Subbaraman and A. R. Teel, "Robustness of recurrence for a class of stochastic hybrid systems," 2015, submitted. 